\title{
Bending Dynamics of Acetylene: New Modes Born in Bifurcations of Normal Modes ${ }^{\dagger}$
}

\author{
Vivian Tyng and Michael E. Kellman* \\ Department of Chemistry, University of Oregon, Eugene, Oregon 97403 \\ Received: December 16, 2005; In Final Form: March 16, 2006
}

(W) This paper contains enhanced objects available on the Internet at http://pubs.acs.org/jpcbfk.

\begin{abstract}
Semiclassical techniques are used to analyze highly excited pure bending vibrational dynamics from spectra of $\mathrm{C}_{2} \mathrm{H}_{2}$. An analytic bifurcation approach is developed, based on critical points of a classical version of the quantum fitting Hamiltonian. At high energy four new types of anharmonic modes are born in bifurcations of the normal modes: local, orthogonal, precessional, and counter-rotator. Visual insight into their nature is obtained with the help of computer-generated three-dimensional animations. The connection between the local mode and the acetylene-vinylidene isomerization "reaction mode" is considered.
\end{abstract}

\section{Introduction}

At low energies, near the minimum in the potential energy surface, the internal vibrational motions of molecules are described by the normal modes model. At higher energy, due to the interplay of anharmonicity and resonance couplings, the normal modes model breaks down. The description of the vibrational motions, including motions involved in molecular rearrangements, is then an outstanding problem.

We have been developing an approach ${ }^{1-13}$ that analyzes the classical version of the effective quantum Hamiltonian used to fit experimental spectra, to get knowledge of "new modes" that describe the motion at high energy. These new modes are born in bifurcations, or branchings associated with the original normal modes.

Our approach to the bifurcation problem takes advantage of simplifications s, $^{2,10,14}$ connected with the polyad number, or total vibrational quantum number of the fitting Hamiltonian. The normal modes and associated modes born in bifurcations can be determined analytically by solution for the critical points of the fitting Hamiltonian, suitably reduced in phase space dimension by means of the polyad number. There is no need for numerical integration of Hamilton's equations or searches by means of surfaces of section in high-dimensional phase spaces. This approach has been successfully applied to systems of increasing complexity, including triatomics with multiple Fermi resonances and classical chaos. ${ }^{9,10,14}$

The subject of this paper is the systematic analysis of pure bending spectra of acetylene using the analytical critical points method, for the extensively measured spectra with $l=0$ vibrational angular momentum. From the spectroscopic fitting Hamiltonian, ${ }^{15}$ it is known that the pure bend dynamics are approximately decoupled from the stretches. This makes for a very interesting system that represents a new level of complexity for a molecular application of the analytical critical points method and a notable way station along the road to analysis of the full stretch-bend vibrational dynamics. Acetylene dynamics including the rearrangement to the vinylidene isomer are of great interest because of their importance in combustion. ${ }^{16}$

* To whom correspondence may be addressed. E-mail: kellman@uoregon.edu.

† Part of the special issue "Robert J. Silbey Festschrift".
We have several specific aims in performing the bifurcation analysis of the acetylene bends system. (1) To obtain a systematic global analysis of the bifurcations that lead to novel modes. Can we understand the number and character of the new modes and the role of each in the quantum dynamics? Is there a unique "evolutionary tree" of new modes, born in bifurcations of the normal modes? Or are there bifurcations disconnected from the normal mode "tree" (due to saddle node bifurcations, as observed in an earlier analysis of $\mathrm{HCP}^{11}$ )? (2) To develop computer animations to depict the new modes in a way that immediately brings out their physical meaning. (3) To assess the prospects for further extension of the analytical bifurcation approach to larger systems.

The dynamics of a classical version of the spectroscopic fitting Hamiltonian for $l=0$ have been extensively studied by Taylor and co-workers. ${ }^{17,18}$ By numerical integration of Hamilton's equation at two different values of the polyad number, they found evidence for several novel types of modes at high energy. The analytical bifurcation analysis of the present paper employs methods complementary to those of refs 17 and 18 . We will seek to uncover further regularities in their important observations and further systematize the global picture of the acetylene bend dynamics.

\section{Spectroscopic Hamiltonian of $\mathrm{C}_{2} \mathrm{H}_{2}$ Bends}

In this section we first describe the quantum fitting Hamiltonian and then describe the corresponding semiclassical Hamiltonian for which we will perform the bifurcation analysis in later sections.

A. Quantum Fitting Hamiltonian. The full-dimensional $\mathrm{C}_{2} \mathrm{H}_{2}$ stretch-bend system ${ }^{19}$ has stretch and bend normal modes with quantum numbers $n_{1}, n_{2}, n_{3}, n_{4}, n_{5}$ for the symmetric $\mathrm{C}-\mathrm{H}$ stretch, $\mathrm{C}-\mathrm{C}$ stretch, antisymmetric $\mathrm{C}-\mathrm{H}$ stretch, and trans and cis bend. Both bends are doubly degenerate; therefore two additional quantum numbers $l_{4}, l_{5}$ are introduced to label the vibrational angular momenta associated with $n_{4}, n_{5}\left(\left|l_{i}\right| \leq n_{i}\right)$. The spectra require resonance couplings in the effective Hamiltonian to get a proper fit. These couplings mix the normal mode zero-order states, thereby destroying $n_{i}, l_{i}$ as good quantum numbers, yet they leave three conserved polyad numbers as determined by a "resonance vector" analysis ${ }^{20-22}$ 


$$
\begin{gathered}
N_{\mathrm{t}}=5 n_{1}+3 n_{2}+5 n_{3}+n_{4}+n_{5} \\
N_{\mathrm{s}}=n_{1}+n_{2}+n_{3} \\
l=l_{4}+l_{5}
\end{gathered}
$$

corresponding to total quantum number, stretch quantum number, and vibrational angular momentum, respectively. The inversion symmetry (gerade/ungerade) and parity $( \pm)$ are also conserved. ${ }^{23}$ Each polyad of quantum levels can therefore be labeled as $\left\{N_{\mathrm{t}}, N_{\mathrm{s}}, l\right\}^{g / u, \pm}$. Because the $g / u, \pm$ distinctions are not important in our classical analysis, we focus primarily on the three polyad numbers of (1).

Only zero-order states (hereafter ZOS) belonging to the same polyad $\left\{N_{\mathrm{t}}, N_{\mathrm{s}}, l\right\}$ are mixed by the resonance couplings. A further simplification makes it possible for us to analyze pure bend polyads separately from stretch excitations. As shown in detail elsewhere, ${ }^{8,15}$ the pure bend ZOS are not coupled to states with nonzero stretch quanta by any of the couplings needed to fit the spectrum with the spectroscopic Hamiltonian. Hence, the pure bending states form a subsystem with separate polyads $\left\{N_{\mathrm{t}}, 0, l\right\}$. We designate the pure bending polyads by $\left[N_{\mathrm{b}}, l\right]$ with

$$
N_{\mathrm{b}}=N_{\mathrm{t}}=n_{4}+n_{5}
$$

The spectroscopic fit of Jacobson et al. $^{15}$ is the most appropriate for investigating the bending dynamics. Its parameters were obtained by fitting energy and intensity data, mostly from dispersed fluorescence spectra. There are more recent fits ${ }^{19,24}$ for the full stretch-bend system. However, most of the states they include have only moderate excitation in the bending modes despite their overall high vibrational energy. Hence the fit of ref 15 is expected to give the best available representation of the bending dynamics. In section 6 , we will examine how the bifurcation analysis of these various Hamiltonians compare.

The spectroscopic Hamiltonian specialized to the pure bends system $^{15}$ contains a normal-mode zero-order part plus three coupling terms

$$
\hat{H}=\hat{H}_{0}+\hat{V}_{\mathrm{DDI}}+\hat{V}_{l}+\hat{V}_{\mathrm{DDII}}
$$

The zero-order part has diagonal terms

$$
\begin{array}{r}
\hat{H}_{0}=\sum_{i=4}^{5} \omega_{i} n_{i}+\sum_{i, j=4 ; i \leq j}^{5} x_{i j} n_{i} n_{j}+\sum_{i, j, k=4 ; i \leq j \leq k}^{5} y_{i j k} n_{i} n_{j} n_{k}+ \\
\sum_{i, j=4 ; i \leq j}^{5} g_{i j} l_{i} l_{j}
\end{array}
$$

and three resonance couplings which can be written as

\section{Darling-Dennison I (DD-I) resonance}

$$
\begin{aligned}
&\left\langle n_{4}{ }^{l_{4}}, n_{5}{ }^{l_{5}}\left|\hat{V}_{\mathrm{DDI}}\right|\left(n_{4}-2\right)^{l_{4}},\left(n_{5}+2\right)^{l_{5}}\right\rangle=\frac{S_{45}}{4}\left[( n _ { 4 } { } ^ { 2 } - l _ { 4 } { } ^ { 2 } ) \left(n_{5}+\right.\right. \\
&\left.\left.l_{5}+2\right)\left(n_{5}-l_{5}+2\right)\right]^{1 / 2}
\end{aligned}
$$

\section{2. $l$-resonance}

$$
\begin{array}{r}
\left\langle n_{4}{ }^{l_{4}}, n_{5}{ }^{l_{5}}\left|\hat{V}_{l}\right| n_{4}{ }^{\left(l_{4} \pm 2\right)}, n_{5}{ }^{\left(l_{5} \mp 2\right)}\right\rangle= \\
\frac{R_{45}}{4}\left[\left(n_{4} \mp l_{4}\right)\left(n_{4} \pm l_{4}+2\right) \times\right. \\
\left.\left(n_{5} \pm l_{5}\right)\left(n_{5} \mp l_{5}+2\right)\right]^{1 / 2}
\end{array}
$$

3. Darling-Dennison II (DD-II) resonance

$$
\begin{array}{r}
\left\langle n_{4}^{l_{4}}, n_{5}{ }^{l_{5}}\left|\hat{V}_{\mathrm{DDII}}\right|\left(n_{4}-2\right)^{\left(l_{4} \mp 2\right)}\left(n_{5}+2\right)^{\left(l_{5} \pm 2\right)}\right\rangle=\frac{R_{45}+2 g_{45}}{16} \times \\
{\left[\left(n_{4} \pm l_{4}\right)\left(n_{4} \pm l_{4}-2\right)\left(n_{5} \pm l_{5}+2\right)\left(n_{5} \pm l_{5}+4\right)\right]^{1 / 2}}
\end{array}
$$

where $R_{45}=r_{45}{ }^{0}+r_{445}\left(n_{4}-1\right)+r_{455}\left(n_{5}-1\right)$.

As depicted in Figure 1, $\hat{V}_{\text {DDI }}$ couples ZOS into columns with the same $\left(l_{4}, l_{5}\right)$, while $\hat{V}_{l}$ couples them into rows with the same $\left(n_{4}, n_{5}\right)$. The $\hat{V}_{\text {DDII }}$ resonance is actually composed of two couplings that run in diagonal directions in Figure 1, so all ZOS in a polyad are connected in a network. The Hamiltonian containing these three resonances is therefore not separable.

Alternatively, $\hat{H}$ can be expressed in terms of raising and lowering operators acting on the two isotropic two-dimensional oscillators corresponding to the trans and cis bends. The symmetry-adapted operators are $\hat{a}_{4 d^{\dagger}}, \hat{a}_{4 g^{\dagger}}, \hat{a}_{4 d}, \hat{a}_{4 g}$, and $\hat{a}_{5 d^{\dagger}}$, $\hat{a}_{5 g}^{\dagger}, \hat{a}_{5 d}, \hat{a}_{5 g}$, respectively ${ }^{25}$

$$
\begin{gathered}
\left.\hat{a}_{i d}^{\dagger}\left|n_{i}^{\left.l_{i}\right\rangle}=\sqrt{\frac{n_{i}+l_{i}+2}{2}}\right|\left(n_{i}+1\right)^{\left(l_{i}+1\right)}\right\rangle \\
\hat{a}_{i g}^{\dagger}\left|n_{i}^{l_{i}}\right\rangle=\sqrt{\frac{n_{i}-l_{i}+2}{2}}\left|\left(n_{i}+1\right)^{\left(l_{i}-1\right)}\right\rangle \\
\hat{a}_{i d}\left|n_{i}^{l_{i}}\right\rangle=\sqrt{\frac{n_{i}+l_{i}}{2}}\left|\left(n_{i}-1\right)^{\left(l_{i}-1\right)}\right\rangle \\
\hat{a}_{i g}\left|n_{i}^{l_{i}}\right\rangle=\sqrt{\frac{n_{i}-l_{i}}{2}}\left|\left(n_{i}-1\right)^{\left(l_{i}+1\right)}\right\rangle
\end{gathered}
$$

for $i=4,5$.

The resonance terms can be expressed as

$$
\begin{gathered}
\hat{V}_{\mathrm{DDI}}=S_{45}\left(\hat{a}_{4 d}^{\dagger} \hat{a}_{4 g}^{\dagger} \hat{a}_{5 d} \hat{a}_{5 g}+\hat{a}_{4 d} \hat{a}_{4 g} \hat{a}_{5 d}^{\dagger} \hat{a}_{5 g}^{\dagger}\right) \\
\hat{V}_{l}=R_{45}\left(\hat{a}_{4 d} \hat{a}_{4 g}^{\dagger} \hat{a}_{5 d}^{\dagger} \hat{a}_{5 g}+\hat{a}_{4 d}^{\dagger} \hat{a}_{4 g} \hat{a}_{5 d} \hat{a}_{5 g}^{\dagger}\right) \\
\hat{V}_{\mathrm{DDII}}=\frac{R_{45}+2 g_{45}}{4}\left(\hat{a}_{4 d}^{\dagger} \hat{a}_{4 d}^{\dagger} \hat{a}_{5 d} \hat{a}_{5 d}+\hat{a}_{4 g}^{\dagger} \hat{a}_{4 g}^{\dagger} \hat{a}_{5 g} \hat{a}_{5 g}+\right. \\
\left.\hat{a}_{4 d} \hat{a}_{4 d} \hat{a}_{5 d}^{\dagger} \hat{a}_{5 d}^{\dagger}+\hat{a}_{4 g} \hat{a}_{4 g} \hat{a}_{5 g}^{\dagger} \hat{a}_{5 g}^{\dagger}\right)
\end{gathered}
$$

B. Semiclassical Correspondence. The next step is to transform $\hat{H}$ into a semiclassical Hamiltonian. For this purpose one applies the Heisenberg correspondence principle $26-28$

$$
\hat{a}_{i} \rightarrow \sqrt{I_{i}} \mathrm{e}^{-i \phi_{i}}, \quad \hat{a}_{i}^{\dagger} \rightarrow \sqrt{I_{i}} \mathrm{e}^{i \phi_{i}}
$$

for $i=4 d, 4 g, 5 d$, and $5 g$. Canonically conjugate action-angle variables in the classical Hamiltonian system are $\left(I_{i}, \phi_{i}\right)$ with

$$
\begin{aligned}
& I_{4 d}=\left(n_{4}+1+l_{4}\right) / 2 \\
& I_{4 g}=\left(n_{4}+1-l_{4}\right) / 2 \\
& I_{5 d}=\left(n_{5}+1+l_{5}\right) / 2 \\
& I_{5 g}=\left(n_{5}+1-l_{5}\right) / 2
\end{aligned}
$$

A simplification becomes possible because there exist two conserved classical actions corresponding to the two quantum polyad numbers

$$
I_{4 d}+I_{4 g}+I_{5 d}+I_{5 g}=n_{4}+n_{5}+2=N_{\mathrm{b}}+2
$$




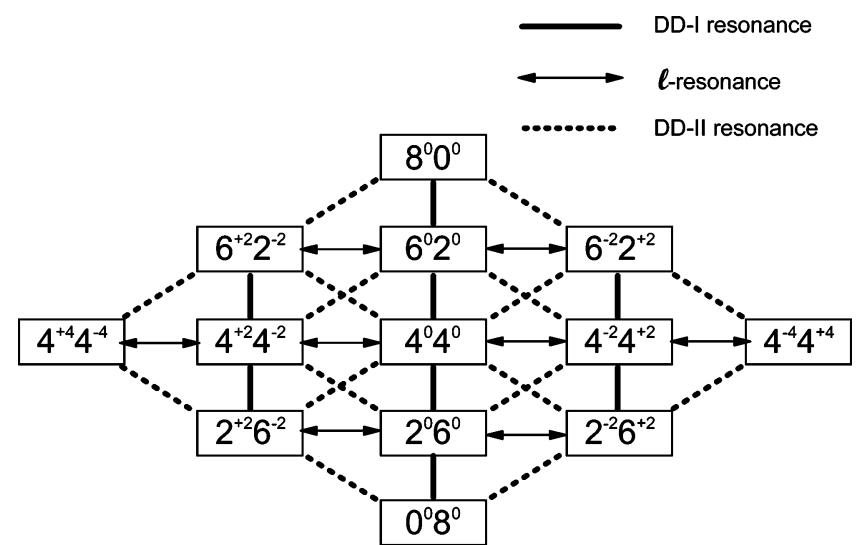

Figure 1. Structure of zero-order states and couplings for the polyad $\left[N_{b}, l\right]^{g / u}=[8,0]^{g}$. The DD-I, $l$-resonance, and DD-II couplings are indicated as lines connecting different pairs of zero order normal mode states with quantum numbers $\left(n_{4}{ }^{14}, n_{5}{ }^{15}\right)$.

$$
I_{4 d}-I_{4 g}+I_{5 d}-I_{5 g}=l_{4}+l_{5}=l
$$

A useful canonical transformation then gives new variables

$$
\begin{gathered}
K_{a}=\left(I_{4 d}+I_{4 g}+I_{5 d}+I_{5 g}\right) / 2=\left(n_{4}+n_{5}+2\right) / 2 \\
K_{b}=\left(I_{4 d}-I_{4 g}+I_{5 d}-I_{5 g}\right) / 2=\left(l_{4}+l_{5}\right) / 2 \\
J_{a}=\left(I_{4 d}+I_{4 g}-I_{5 d}-I_{5 g}\right) / 2=\left(n_{4}-n_{5}\right) / 2 \\
J_{b}=-\left(I_{4 d}-I_{4 g}-I_{5 d}+I_{5 g}\right) / 2=-\left(l_{4}-l_{5}\right) / 2 \\
\theta_{a}=\left(\phi_{4 d}+\phi_{4 g}+\phi_{5 d}+\phi_{5 g}\right) / 2 \\
\theta_{b}=\left(\phi_{4 d}-\phi_{4 g}+\phi_{5 d}-\phi_{5 g}\right) / 2 \\
\psi_{a}=\left(\phi_{4 d}+\phi_{4 g}-\phi_{5 d}-\phi_{5 g}\right) / 2 \\
\psi_{b}=-\left(\phi_{4 d}-\phi_{4 g}-\phi_{5 d}+\phi_{5 g}\right) / 2
\end{gathered}
$$

Of the new actions and their conjugate angles $\left(K_{a}, \theta_{a}\right),\left(K_{b}, \theta_{b}\right)$, $\left(J_{a}, \psi_{a}\right)$, and $\left(J_{b}, \psi_{b}\right)$, the actions $K_{a}$ and $K_{b}$ are conserved. Their conjugate angles $\theta_{a}, \theta_{b}$ are cyclic variables that do not appear explicitly in the Hamiltonian.

Compared to the notation of ref 17, our actions are multiplied by a factor of 2, while the angles are reduced by a factor of $1 / 2$. Computationally this difference is inconsequential, but our definition has an advantage for explaining the topology of the classical phase space, as will be explained in section IV.A.

The resulting classical Hamiltonian for arbitrary $l$ is

$$
H^{l}\left(K_{a}, K_{b}, J_{a}, J_{b}, \psi_{a}, \psi_{b}\right)=H_{0}+V_{\mathrm{DDI}}+V_{l}+V_{\mathrm{DDII}}+V_{\mathrm{DDII}}{ }^{\prime}
$$

with

$$
\begin{gathered}
H_{0}=\omega_{4}\left(K_{a}+J_{a}-1\right)+\omega_{5}\left(K_{a}-J_{a}-1\right)+x_{44}\left(K_{a}+J_{a}-\right. \\
1)^{2}+x_{45}\left(K_{a}+J_{a}-1\right)\left(K_{a}-J_{a}-1\right)+x_{55}\left(K_{a}-J_{a}-\right. \\
1)^{2}+y_{444}\left(K_{a}+J_{a}-1\right)^{3}+y_{445}\left(K_{a}+J_{a}-1\right)^{2}\left(K_{a}-J_{a}-\right. \\
1)+y_{455}\left(K_{a}+J_{a}-1\right)\left(K_{a}-J_{a}-1\right)^{2}+y_{555}\left(K_{a}-J_{a}-\right. \\
1)^{3}+g_{44}\left(K_{b}-J_{\mathrm{b}}\right)^{2}+g_{45}\left(K_{b}^{2}-J_{b}^{2}\right)+g_{55}\left(K_{b}+J_{b}\right)^{2}
\end{gathered}
$$

$$
\begin{gathered}
V_{\mathrm{DDI}}=\frac{S_{45}}{2}\left[\left(K_{a}^{2}-K_{b}^{2}\right)^{2}+\left(J_{a}^{2}-J_{b}^{2}\right)^{2}-2\left(K_{a}^{2}+K_{b}^{2}\right)\right. \\
\left.\left(J_{a}^{2}+J_{b}^{2}\right)-8 K_{a} K_{b} J_{a} J_{b}\right]^{1 / 2} \cos \left[2 \psi_{a}\right] \\
V_{l}=\frac{R_{45}}{2}\left[\left(K_{a}^{2}-K_{b}^{2}\right)^{2}+\left(J_{a}^{2}-J_{b}^{2}\right)^{2}-2\left(K_{a}^{2}+K_{b}^{2}\right)\left(J_{a}^{2}+\right.\right. \\
\left.\left.J_{b}^{2}\right)-8 K_{a} K_{b} J_{a} J_{b}\right]^{1 / 2} \cos \left[2 \psi_{b}\right] \\
V_{\mathrm{DDII}}=\frac{R_{45}+2 g_{45}}{8}\left[\left(K_{a}+K_{b}\right)^{2}-\left(J_{a}-J_{b}\right)^{2}\right] \cos \left[2 \left(\psi_{a}-\right.\right. \\
V_{\mathrm{DDII}}{ }^{\prime}=\frac{R_{45}+2 g_{45}}{8}\left[\left(K_{a}-K_{b}\right)^{2}-\left(J_{a}+J_{b}\right)^{2}\right] \cos \left[2 \left(\psi_{a}+\right.\right.
\end{gathered}
$$$$
\left.\left.\psi_{b}\right)\right]
$$

where $V_{\text {DDII }}, V_{\text {DDII }}$ correspond to the two diagonal directions of the quantum coupling $\hat{V}_{\text {DDII }}$ mentioned above in regard to Figure 1; and with $R_{45}=r_{45}{ }^{0}+r_{445}\left(K_{a}+J_{a}-2\right)+r_{455}\left(K_{a}-\right.$ $J_{a}-2$ ).

Within each polyad the conserved $K_{a}, K_{b}$ are held fixed. Eliminating the conjugate cyclic angles $\theta_{a}, \theta_{b}$ because they play a trivial role in the dynamics, the reduced phase space is fourdimensional. The remaining variables $J_{a}, J_{b}, \psi_{a}, \psi_{b}$ evolve according to Hamilton's equations of motion

$$
\begin{gathered}
\dot{J}_{a}=-\frac{\partial H}{\partial \psi_{a}} \\
\dot{J}_{b}=-\frac{\partial H}{\partial \psi_{b}} \\
\dot{\psi}_{a}=\frac{\partial H}{\partial J_{a}} \\
\dot{\psi}_{b}=\frac{\partial H}{\partial J_{b}}
\end{gathered}
$$

For the case with zero total vibrational angular momentum ( $l$ $=0, K_{b}=l / 2=0$ ), the Hamiltonian (13) can be simplified to

$$
H=H_{l=0}=H_{0}+V_{\mathrm{DDI}}+V_{l}+V_{\mathrm{DDII}}+V_{\mathrm{DDII}}^{\prime}
$$

with

$$
\begin{array}{r}
H_{0}=\omega_{4}\left(K_{a}+J_{a}-1\right)+\omega_{5}\left(K_{a}-J_{a}-1\right)+x_{44}\left(K_{a}+J_{a}-\right. \\
1)^{2}+x_{45}\left(K_{a}+J_{a}-1\right)\left(K_{a}-J_{a}-1\right)+x_{55}\left(K_{a}-J_{a}-\right. \\
1)^{2}+y_{444}\left(K_{a}+J_{a}-1\right)^{3}+y_{445}\left(K_{a}+J_{a}-1\right)^{2}\left(K_{a}-J_{a}-\right. \\
1)+y_{455}\left(K_{a}+J_{a}-1\right)\left(K_{a}-J_{a}-1\right)^{2}+y_{555}\left(K_{a}-J_{a}-\right. \\
1)^{3}+\left(g_{44}-g_{45}+g_{55}\right) J_{b}^{2}
\end{array}
$$

$$
\begin{array}{r}
V_{\mathrm{DDI}}=\frac{S_{45}}{2}\left[K_{a}^{4}+\left(J_{a}{ }^{2}-J_{b}{ }^{2}\right)^{2}-2 K_{a}^{2}\left(J_{a}{ }^{2}+J_{b}{ }^{2}\right)\right]^{1 / 2} \cos \left[2 \psi_{a}\right] \\
=S_{1}\left(K_{a}, J_{a}, J_{b}\right) \cos \left[2 \psi_{a}\right] \\
V_{l}=\frac{R_{45}}{2}\left[K_{a}^{4}+\left(J_{a}{ }^{2}-J_{b}{ }^{2}\right)^{2}-2 K_{a}{ }^{2}\left(J_{a}{ }^{2}+J_{b}{ }^{2}\right)\right]^{1 / 2} \cos \left[2 \psi_{b}\right] \\
=S_{2}\left(K_{a}, J_{a}, J_{b}\right) \cos \left[2 \psi_{b}\right]
\end{array}
$$




$$
\begin{aligned}
& V_{\mathrm{DDII}}=\frac{R_{45}+2 g_{45}}{8}\left[K_{a}^{2}-\left(J_{a}-J_{b}\right)^{2}\right] \cos \left[2\left(\psi_{a}-\psi_{b}\right)\right] \\
& =S_{3}\left(K_{a}, J_{a}, J_{b}\right) \cos \left[2\left(\psi_{a}-\psi_{b}\right)\right] \\
& V_{\text {DDII }}{ }^{\prime}=\frac{R_{45}+2 g_{45}}{8}\left[K_{a}^{2}-\left(J_{a}+J_{b}\right)^{2}\right] \cos \left[2\left(\psi_{a}+\psi_{b}\right)\right] \\
& =S_{4}\left(K_{a}, J_{a}, J_{b}\right) \cos \left[2\left(\psi_{a}+\psi_{b}\right)\right]
\end{aligned}
$$

We now proceed to the bifurcation analysis of this Hamiltonian.

\section{Analytically Scalable Polyad Bifurcation Analysis}

In this section we discuss important general features of the method of the critical points bifurcation analysis, in particular its analytic and scalable character; in the next section, we present details of implementation.

The Hamiltonian in (18) is a nonintegrable system. In general, the dynamics of such a system can only be understood by numerical integration of Hamilton's equations and subsequent analysis, e.g., with sections in phase space. This is possible for the system here, but with increasing degrees of freedom, it will quickly become intractable. We therefore pursue a method with the potential to be extended to larger systems.

We want to take advantage of the fact that the polyad Hamiltonian affords computation of the large-scale phase space structure by analytic means, i.e., by solution of simple algebraic equations related to the Hamiltonian function, rather than numerical solution of the equations of motion. Specifically, we seek the critical points of the polyad Hamiltonian, defined in a phase space naturally reduced in dimension by means of the conserved polyad numbers. In the case of acetylene bends treated here, the critical points give periodic orbits (POs) or "vibrational modes" when the Hamiltonian is expanded back to the full phase space, including polyad numbers and their conjugate angles. The critical points are solutions of algebraic equations involving polynomials and trigonometric functions. Because they are obtained analytically, it is not necessary to perform numerical integration of Hamilton's equation and analysis of surfaces of section. This is expected to become extremely advantageous as the number of degrees of freedom and phase space dimensions increase.

We call the modes obtained by the analytic critical points method the "primary" modes of the system. We use this terminology because the primary modes are the lowest period orbits consistent with the polyad structure. Hence, they are expected to organize the large-scale structure of the molecular phase space. We will see that the structure of the Hamiltonian severely limits the number and character of the primary modes, and even their location in phase space. (As discussed later, in more general systems, the critical points may correspond to higher-dimensional invariant tori rather than one-dimensional modes, but these structures are still expected to organize the phase space.) The critical points approach has been developed and applied to systems with two ${ }^{5-8,11-13,29-31}$ and three $e^{9,10,14}$ coupled modes. In the exposition here we build up from these simpler systems to make the generalization to the acetylene bends system as clear as possible.

First, consider a two-mode system with a single resonance coupling and a conserved polyad number $I$ conjugate to the cyclic variable $\theta$

$$
H=H_{0}(I, J)+V(I, J, \psi)
$$

with Poisson bracket

$$
\{H, I\}=0
$$

Regarding the polyad number $I$ as a fixed parameter, this Hamiltonian is a function in the reduced phase space with variables $(J, \psi)$. According to Hamilton's equations, the critical points of this Hamiltonian satisfy

$$
\dot{J}=0, \quad \dot{\psi}=0
$$

The physical meaning of a critical point $\left(J_{0}, \psi_{0}\right)$ at polyad $I_{0}$ is seen by considering the full phase space with canonical coordinates $(I, \theta, J, \psi)$. Only the cyclic variable $\theta$ varies with time, with a constant frequency $(\partial H / \partial I)_{\mathrm{I}_{0}, \psi_{0}}$. Therefore, the critical point in the reduced phase space corresponds to a PO in the full phase space. This might be one of the low-energy normal modes of the system, but with the presence of the resonance coupling in the Hamiltonian, it might be a new mode born in a bifurcation.

This analysis easily generalizes ${ }^{2,10}$ to a nonintegrable Hamiltonian with $N$ degrees of freedom and $N-1$ independent resonances, so that there is a conserved polyad number. This has been exploited to analyze the bifurcations of the $\mathrm{H}_{2} \mathrm{O}$ spectroscopic Hamiltonian and assign quantum spectra. ${ }^{9}, 10,14,32$

Here we generalize the analysis further to include systems with more than one polyad number. This is of concern for us, because the acetylene stretch-bend Hamiltonian has three polyad numbers ${ }^{20,21,33}$ (see eq 1); the pure bends system has two polyad numbers, $N_{\mathrm{b}}$ and $l$. Consider then an $N$-mode Hamiltonian with $M$ polyad numbers

$$
H=H_{0}\left(I_{i}, J_{k}\right)+V\left(I_{i}, J_{k}, \psi_{k}\right)
$$

with $(i=1,2, \ldots, M ; k=M+1, \ldots, N)$. A critical point in the reduced phase space $\left(J_{k}, \psi_{k}\right)$ satisfies

$$
\dot{J}=0, \quad \dot{\psi}_{k}=0 \text { for } k=M+1, \ldots, N
$$

It has up to $M$ nonzero cyclic frequencies in the full phase space: $\left(\dot{\theta}_{1}, \ldots, \dot{\theta}_{M}\right)$. When the number of polyad numbers is $M$ $=1$, this gives a closed PO, one of the primary modes.

When the number of polyad numbers is $M>1$ and none of the $M$ frequencies is commensurate, the full trajectory in general comprises motion on an $M$-dimensional torus, defined by the $M$ angles conjugate to the polyad numbers. This is a situation not to our knowledge analyzed previously in a molecular setting; it will have real implications for the bifurcation analysis of full stretch-bend acetylene dynamics, as discussed briefly in the closing section.

In the $\mathrm{C}_{2} \mathrm{H}_{2}$ pure bending system with polyad numbers $N_{b}=$ $2 K_{a}-2, l=2 K_{b}$, a critical point in the reduced phase space corresponds, except in special cases, to motion on a twodimensional invariant torus in the full phase space. The torus is parametrized by $\theta_{a}, \theta_{b}$. The motion is typically quasi-periodic since the frequencies $\dot{\theta}_{a}$ and $\dot{\theta}_{b}$ are usually noncommensurate. This situation will in fact arise and be very important in the full stretch-bend acetylene dynamics, as discussed briefly in the concluding section.

However, in the general case of systems with $M>1$, there are special cases when (1) one or more of the cyclic angles has zero frequency, (2) some of the frequencies are locked in integer ratios (commensurate) for special physical reasons, or (3) the torus thickness goes to zero for special values of the actions (this possibility does not pertain to the problem here with vibrational angular momenta $l_{4}, l_{5}$ ). In these cases the full trajectory of a critical point has fewer than $M$ independent frequencies. The $l=0$ polyad series analyzed here forms a 


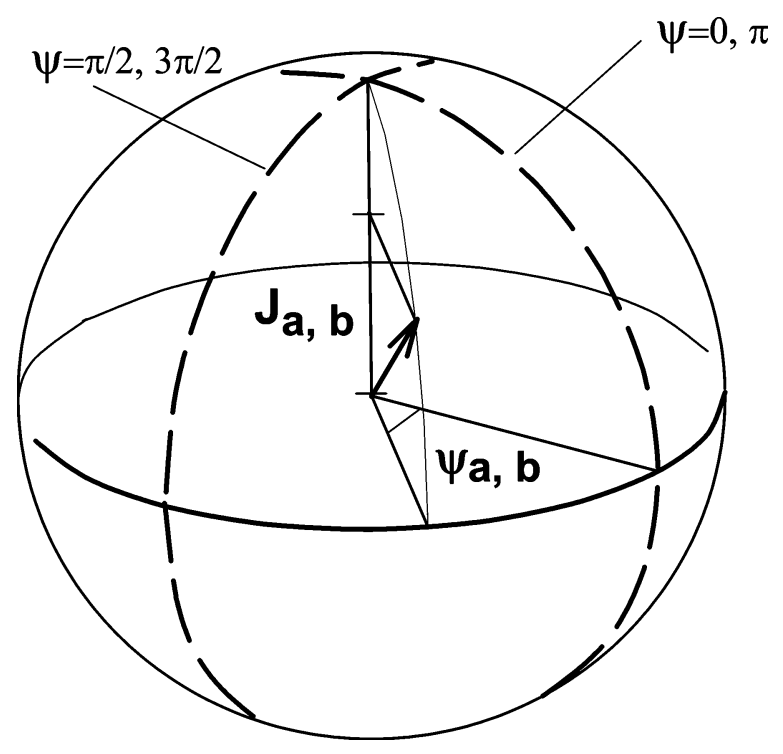

Figure 2. Coordinates for the phase space spheres. There are two spheres, one for the phase space variables $J_{a}, \psi_{a}$ and one for the variables $J_{b}, \psi_{b}$, see (12) and following for definitions. Each sphere has its own $J_{i}, \psi_{i}$. For each sphere, the great circles on which critical points lie are indicated by dashed lines, at the indicated values of $\psi=\psi_{a}$ or $\psi_{b}$.

special case for type 1 . As described in the following sections, all except two physically unimportant critical points in this series turn out to have

$$
\dot{\theta}_{b}=0
$$

so the full trajectories of these critical points reduce to closed POs parametrized by $\theta_{a}$. Hence they are either normal modes or new anharmonic modes born in bifurcations.

In the remainder of this paper, we shall be concerned with the pure bends system, with conserved polyad numbers $N_{b}=$ $2 K_{a}-2, l=2 K_{b}=0$. In future work we will consider the interesting problem of dynamics of systems with $l \neq 0$ or stretch excitation, where bifurcations of the normal modes do not in general give new periodic orbits but rather higher-dimensional tori.

\section{Implementation of the Bifurcation Analysis}

We consider first the search for the critical points and then the analysis of their stability character.

A. Search for the Critical Points. In our system, with conserved polyad actions $K_{a}, K_{b}$ and conjugate angles $\theta_{a}, \theta_{b}$, the dynamics of the reduced Hamiltonian are contained in the behavior of the phase space variables $\left(J_{a}, J_{b}, \psi_{a}, \psi_{b}\right)$. This can be represented in constant energy surfaces of section mapped on two spherical phase space surfaces, one a "DD-I sphere" with variables $J_{a}, \psi_{\mathrm{a}}$ and the other an " $l$-resonance sphere" with variables $J_{b}, \psi_{b}$. The allowed phase space for a given energy partially or fully covers the phase space spheres. The phase space spheres and their coordinates are illustrated in Figure 2. (These phase space spheres are constant energy surfaces of section and should not be confused with the related, but distinct, "polyad phase sphere" 5,8 with constant polyad number rather than constant energy. The latter applies in a variety of contexts including coupled oscillators, dynamical "self-trapping", 34,35 Bose-Einstein condensates, ${ }^{36}$ and other seemingly unrelated applications.)

The critical points of the reduced Hamiltonian are solutions of Hamilton's equations that satisfy

$$
\begin{gathered}
\dot{J}_{a}=-\frac{\partial H}{\partial \psi_{a}}=0 \\
\dot{J}_{b}=-\frac{\partial H}{\partial \psi_{b}}=0 \\
\dot{\psi}_{a}=\frac{\partial H}{\partial J_{a}}=0 \\
\dot{\psi}_{b}=\frac{\partial H}{\partial J_{b}}=0
\end{gathered}
$$

Substituting the classical Hamiltonian from (18), the first two of these equations have the form

$$
\begin{aligned}
&-\frac{\partial H}{\partial \psi_{a}}=2 S_{1} \sin \left[2 \psi_{a}\right]+2 S_{3} \sin \left[2\left(\psi_{a}-\psi_{b}\right)\right] \\
&+ 2 S_{4} \sin \left[2\left(\psi_{a}+\psi_{b}\right)\right]=0 \\
&-\frac{\partial H}{\partial \psi_{b}}=2 S_{2} \sin \left[2 \psi_{b}\right]-2 S_{3} \sin \left[2\left(\psi_{a}-\psi_{b}\right)\right] \\
&+2 S_{4} \sin \left[2\left(\psi_{a}+\psi_{b}\right)\right]=0
\end{aligned}
$$

The analytical expressions for (27 and 28) are also listed in detail in an unpublished dissertation. ${ }^{37} \mathrm{~A}$ sufficient, but not necessary condition for these two equations to be simultaneously satisfied is

$$
\sin \left[2 \psi_{a}\right]=\sin \left[2 \psi_{b}\right]=0
$$

or

$$
\left(\psi_{a}, \psi_{b}\right)=\left(\frac{m \pi}{2}, \frac{n \pi}{2}\right) \text { with } m, n=0,1,2,3
$$

These sufficiency conditions correspond to great circles on the phase space spheres of Figure 2. It is notable that for single resonance systems, the solutions for the critical points of the polyad Hamiltonian necessarily lie on great circles on the phase space sphere. ${ }^{13}$ An important question is whether this generalizes empirically to systems with more degrees of freedom and multiple resonances, where great circle conditions on the critical points such as (32) are not necessary. Satisfaction of the great circle conditions relates to the primacy of certain individual resonances, since the great circles are defined on phase space spheres whose coordinates are defined by individual resonances, as in Figure 2 for the DD-I and $l$-resonance spheres. Location of critical points on great circles was found empirically for multiresonant triatomic Hamiltonians. ${ }^{10}$ It is not clear a priori that this should generalize to the acetylene bends system, because the DD-II coupling is not linearly independent of the DD-I and $l$-resonance couplings; it is even less clear whether the great circles feature will hold in a future treatment of the full acetylene stretch-bend system, which has a great multiplicity of couplings that are not independent.

For the present bends system, we have done a comprehensive search off the great circles for critical points but found that none exists. The reason is apparently that the DD-II coupling is relatively weak, so the terms in (29 and 30) that mix $\psi_{a}, \psi_{b}$ are small.

Thus, every critical point in the bends system treated here will lie simultaneously on a great circle from each of the spheres. With two circles on each of two spheres, there are four possible 
TABLE 1: Analytical Solutions of Bifurcation Results in Figure $6^{a}$

\begin{tabular}{lll}
\hline \multicolumn{1}{c}{ mode } & $\left(\psi_{a}, \psi_{b}\right)$ & \multicolumn{1}{c}{$J_{a}$} \\
\hline local & $(0,0)$ & $42.3217-0.583914 K_{a}-0.268961\left(16255.7+84.8109 K_{a}-K_{a}^{2}\right)^{1 / 2}$ \\
orthogonal & $(0, \pi / 2)$ & $49.7629-0.49687 K_{a}-0.822458\left(2130.16+65.1370 K_{a}-K_{a}^{2}\right)^{1 / 2}$ \\
precessional & $(\pi / 2,0)$ & $21.8360-0.563978 K_{a}-0.460473\left(-1106.54+186.865 K_{a}-K_{a}^{2}\right)^{1 / 2}$ \\
counter-rotator & $(\pi / 2, \pi / 2)$ & $-36.6246-0.536648 K_{a}+0.634238\left(1237.94+287.074 K_{a}-K_{a}^{2}\right)^{1 / 2}$
\end{tabular}

${ }^{a}$ The values of $J_{a}$ are also constrained by $-K_{a} \leq J_{a} \leq K_{a}$.

combinations of these circles. Furthermore, because of symmetry each critical point is accompanied by another on the opposite side of the circle: $\psi_{a} \leftrightarrow \psi_{a}+\pi$ and $\psi_{b} \leftrightarrow \psi_{b}+\pi$. There are therefore 16 combinations of angles describing four physically distinct (modulo symmetry) possible angular locations of critical points on the great circles-one physically distinct situation for each of the following equations:

$$
\begin{gathered}
\left(\psi_{a}, \psi_{b}\right)=(0,0),(0, \pi),(\pi, 0),(\pi, \pi) \\
\left(\psi_{a}, \psi_{b}\right)=\left(0, \frac{\pi}{2}\right),\left(0, \frac{3 \pi}{2}\right),\left(\pi, \frac{\pi}{2}\right),\left(\pi, \frac{3 \pi}{2}\right) \\
\left(\psi_{a}, \psi_{b}\right)=\left(\frac{\pi}{2}, 0\right),\left(\frac{\pi}{2}, \pi\right),\left(\frac{3 \pi}{2}, 0\right),\left(\frac{3 \pi}{2}, \pi\right) \\
\left(\psi_{a}, \psi_{b}\right)=\left(\frac{\pi}{2}, \frac{\pi}{2}\right),\left(\frac{\pi}{2}, \frac{3 \pi}{2}\right),\left(\frac{3 \pi}{2}, \frac{\pi}{2}\right),\left(\frac{3 \pi}{2}, \frac{3 \pi}{2}\right)
\end{gathered}
$$

The remainder of the critical points problem is to solve the last two equations of (25-28) for the actions $J_{a}, J_{b}$ as the polyad number $N_{\mathrm{b}}$ is continuously varied, holding the angles at the above combinations.

A simplification comes from the observation that with the discrete $\psi_{a}, \psi_{b}$ values, (28) can be rewritten as

$$
\begin{aligned}
& \frac{\partial H}{\partial J_{b}}= J_{b}\left\{2\left(g_{44}-g_{45}+g_{55}\right)-\right. \\
& \frac{\left(K_{a}^{2}+J_{a}^{2}-J_{b}^{2}\right)\left(S_{45} \cos \left[2 \psi_{a}\right]+R_{45} \cos \left[2 \psi_{b}\right]\right)}{\sqrt{K_{a}^{4}+\left(J_{a}^{2}-J_{b}{ }^{2}\right)^{2}-2 K_{a}^{2}\left(J_{a}^{2}+J_{\mathrm{b}}{ }^{2}\right)}}- \\
&\left.\frac{R_{45}+2 g_{45}}{2} \cos \left[2 \psi_{a}\right] \cos \left[2 \psi_{b}\right]\right\}=0
\end{aligned}
$$

For the entire accessible range of $J_{a}, J_{b}$, and when $\psi_{a}, \psi_{b}$ are constrained to values in $(33-36)$, the factor between the curly brackets was numerically verified to be always nonzero. Therefore (28) has the very simple solution

$$
J_{b}=0
$$

After substitution for $J_{b}=0$, the remaining condition (27) becomes an easily solved quadratic equation. The results are listed in Table 1. The detailed meaning of these results is discussed in section 5 in relation to the bifurcation diagram Figure 6; in the remainder of the present section, we outline some technical aspects with the aid of a visually accessible approach to the search and then discuss the stability analysis of the critical points once they have been found.

The solution of $(27,28)$ can be visualized in the following way. If one plots the reduced Hamiltonian as a function of the canonical actions $J_{a}, J_{b}$ while holding $\left(\psi_{a}, \psi_{b}\right)$ fixed at the four physically distinct conditions (33-36), critical points correspond to the flat spots (minima, maxima and saddle points) on these plots. This is a generalization of the "pseudopotential" picture

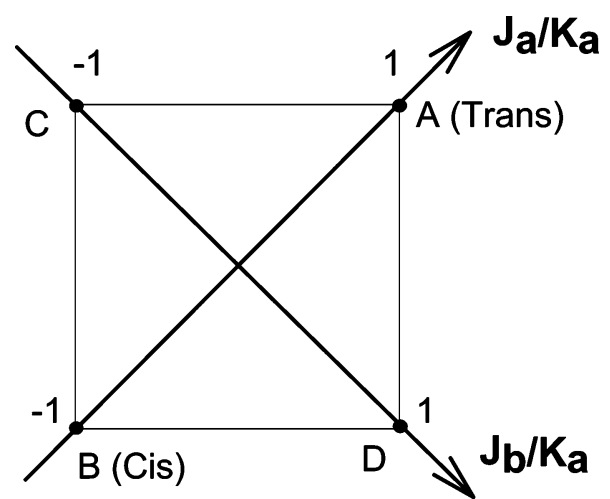

Figure 3. Coordinates of the pseudopotential plots. The scaled coordinates $J_{a} / K_{a}, J_{b} / K_{a}$ show the accessible range of classical actions $J_{a}, J_{b}$ for a given polyad $N_{b}=2 K_{a}-2$. Points $\mathrm{A}, \mathrm{B}, \mathrm{C}$, and D are critical points for all coupling strength, as discussed in section IV.A. $\mathrm{A}$ and $\mathrm{B}$ are the Trans and Cis critical points.

discussed in refs 11 and 38 expanded from a one-dimensional potential to two-dimensional.

Before illustrating this for specific polyads, it is helpful to discuss the coordinates used, with reference to Figure 3 . In the $l=0$ polyads, the values of actions $J_{a}, J_{b}$ are restricted by

$$
\left|J_{a}\right|+\left|J_{\mathrm{b}}\right| \leq K_{a}
$$

In Figures 3 and 4 , we rescale the actions to $J_{a} / K_{a}, J_{b} / K_{a}$ to give the same range $[-1,1]$ for different polyads $N_{b}=2 K_{a}-2$. The points on the boundary of Figure 3 have

$$
\left|J_{a}\right|+\left|J_{b}\right|=K_{a}
$$

Physically, points A, B at the corners correspond to the planar trans and cis bend overtones, and corner points C, D correspond to states with maximum difference in vibrational angular momenta $l_{4}, l_{5}$, e.g.

$$
C: \quad\left|11,,^{11} 11^{-11}\right\rangle, \quad D: \quad\left|11^{-11}, 11^{11}\right\rangle
$$

Points $A-D$ are critical points of the coupled Hamiltonian as well as the zero-order Hamiltonian. The reason is that the couplings vanish at these points. A and B retain their identity as trans and cis normal modes, though they change stability at various bifurcation points. $\mathrm{C}$ and $\mathrm{D}$, however, are not anharmonic modes, but rather are two-tori for states such as those in (41). They never bifurcate. As such, the critical points C and D are trivial for our analysis and are not considered further.

Figure 4 shows pseudopotential plots of the reduced Hamiltonian, on squares with the same coordinates as Figure 3, for the examples of polyad $N_{b}=6$ with $K_{a}=\left(N_{b}+2\right) / 2=4$, and polyad $N_{b}=22$ with $K_{a}=12$. As discussed in section 5 , at $N_{b}$ $=6$ none of the bifurcations has occurred; at $N_{b}=22$ all of the bifurcations have occurred. In Figure $4 \mathrm{a}-\mathrm{d}$ for $N_{b}=6$, the only critical points are at the corners of the four squares, previously labeled $\mathrm{A}-\mathrm{D}$ in Figure 3; the corner points $\mathrm{A}, \mathrm{B}$ on each square correspond to the trans and cis overtones. 
(a)

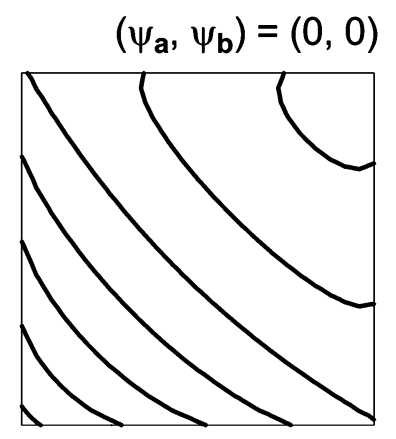

(e)

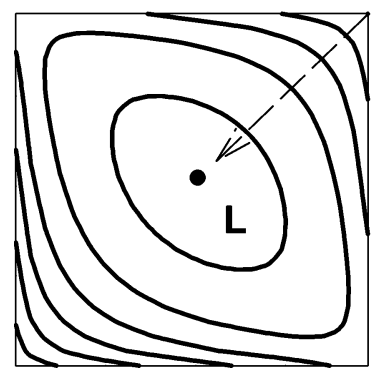

(b)

$(0, \pi / 2)$

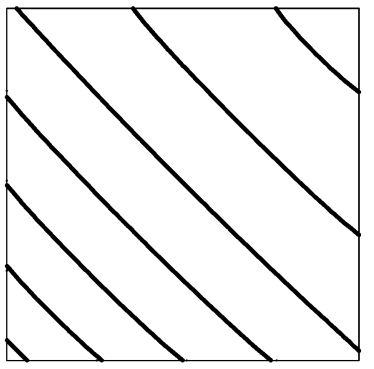

(f)

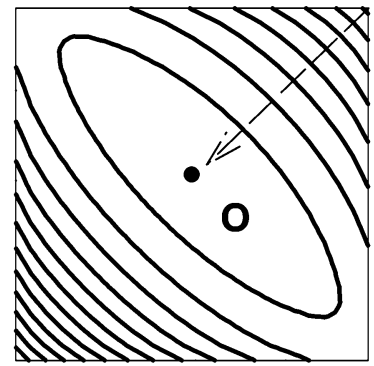

(c)

$(\pi / 2,0)$

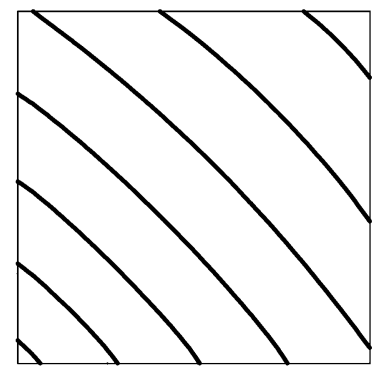

(g)

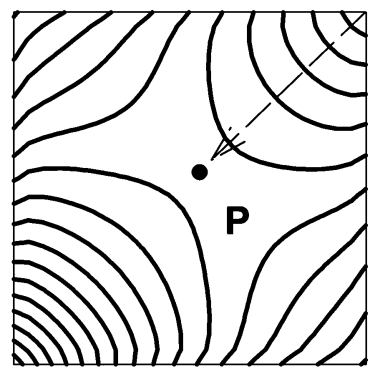

(d)

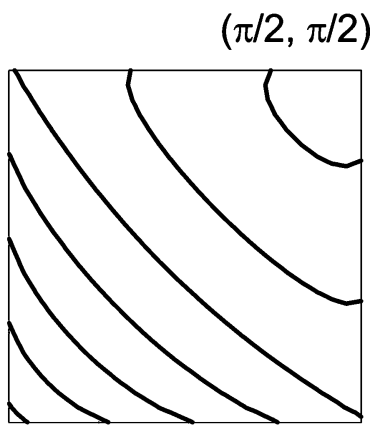

(h)

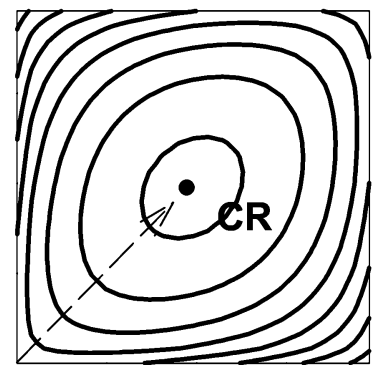

Figure 4. Pseudopotentials of polyads $[6,0]$ and $[22,0]$, given by contour plots, for $\mathrm{H}$ in (18). The squares have the same coordinates as the axes in Figure 3. Panels a-d are for [6,0] with the four values of $\left(\psi_{a}, \psi_{b}\right)$ in $(33-36)$. Panels $\mathrm{e}-\mathrm{h}$ are for [22, 0]. Dark dots in the lower row are new critical points (Local $\mathbf{L}$, Orthogonal $\mathbf{O}$, Precessional $\mathbf{P}$, and Counter-Rotator $\mathbf{C R}$ ) born in bifurcations, after migration from the parent modes (L, $\mathbf{O}, \mathbf{P}$ from Trans and $\mathbf{C R}$ from $\mathbf{C i s}$ ), as indicated by the arrows.

In parts e and f of Figure $4, N_{b}=22$, critical points occur as before at the corners of the four squares. However, there are four new critical points with labels $\mathbf{L}, \mathbf{O}, \mathbf{P}$, and $\mathbf{C R}$ (described in section 5), each in the interior of one of the squares. The new critical points have migrated from the corners along the diagonal of the square, as explained in section 5. Each of these corresponds to a new mode born in a bifurcation from one of the normal modes.

While the graphical pseudopotentials such as Figure 4 are conceptually appealing and will be referred to below, in practice we solve $(27,28)$ analytically for continuously varied $K_{a}$ rather than graphically. On the boundary, the $\left(J_{a}, J_{b}, \psi_{a}, \psi_{b}\right)$ coordinates become inappropriate ("singular") for describing the physical behavior of the system. In particular, when (40) holds, the denominators in (27 and 28) vanish, and $\dot{\psi}_{a}, \dot{\psi}_{b}$ are either infinite (on the edges of Figure 3 except the vertexes, and also the boundaries of the pseudopotential plots in Figure 4) or indeterminate (at the four vertexes, where no unique limit exists). The general definition (25-28) therefore cannot be used to determine whether a boundary point is a critical point or not. A related situation is encountered in ref 13 in the case of single resonance Hamiltonians. In those cases, the angle variable $\psi$ is the azimuthal angle on the respective polyad phase sphere. At the North and South poles of the sphere, this angle is not defined, so the canonical variables $\left(I_{z}, \psi\right)$ are misleading at the poles. A simple example is an ordinary trajectory passing the pole at finite speed. The angular velocity $\dot{\psi}_{i}$ becomes infinite as the pole is approached, but this is entirely an artifact of the coordinates.

To determine the dynamics at the singular points, either direct visualization by running trajectories or a coordinate transformation (called "rotating the sphere" in ref 13) is required. Since direct visualization of four-dimensional phase space is nontrivial, we chose the second path to search for critical points on the boundary. The Hamiltonian (18) is transformed to a local-mode representation using the $x-K$ relationships, ${ }^{39}$ as described in section 7.6 of ref 23. In the local representation, points on the boundary no longer appear "singular" and can be analyzed in a straightforward manner.

B. Stability Analysis of the Critical Points. After locating the critical points, we perform an analysis of their stability character using standard methods of linear analysis. ${ }^{40}$ The interpretation and visualization of the stability analysis are made complicated in comparison with previous systems ${ }^{8}$ by the higher dimensionality of the phase space. After finding a critical point at a certain set of $\left(J_{a}, J_{b}, \psi_{a}, \psi_{b}\right)$, motion in its neighborhood can be linearized as

$$
\begin{aligned}
& \frac{\mathrm{d}}{\mathrm{d} t}\left(\begin{array}{l}
\delta J_{a} \\
\delta J_{b} \\
\delta \psi_{a} \\
\delta \psi_{b}
\end{array}\right)=\left(\begin{array}{llll}
0 & 0 & -1 & 0 \\
0 & 0 & 0 & -1 \\
1 & 0 & 0 & 0 \\
0 & 1 & 0 & 0
\end{array}\right)\left(\begin{array}{llll}
H_{J_{a} J_{a}} & H_{J_{a} J_{b}} & H_{J_{a} \psi_{a}} & H_{J_{a} \psi_{b}} \\
H_{J_{b} J_{a}} & H_{J_{b} J_{b}} & H_{J_{b} \psi_{a}} & H_{J_{b} \psi_{b}} \\
H_{\psi_{a} J_{a}} & H_{\psi_{a} J_{b}} & H_{\psi_{a} \psi_{a}} & H_{\psi_{a} \psi_{b}} \\
H_{\psi_{b} J_{a}} & H_{\psi_{b} J_{b}} & H_{\psi_{b} \psi_{a}} & H_{\psi_{b} \psi_{b}}
\end{array}\right) \\
& \left(\begin{array}{l}
\delta J_{a} \\
\delta J_{b} \\
\delta \psi_{a} \\
\delta \psi_{b}
\end{array}\right)=M\left(\begin{array}{l}
\delta J_{a} \\
\delta J_{b} \\
\delta \psi_{a} \\
\delta \psi_{b}
\end{array}\right)
\end{aligned}
$$

where $H_{x y}=\left(\partial^{2} H / \partial x \partial y\right)$.

The linear stability reflects the time evolution of $\left(\delta J_{a}, \delta J_{b}\right.$, $\left.\delta \psi_{a}, \delta \psi_{b}\right)$, which is determined by the eigenvalues $\lambda_{i}$ of the matrix $M$ above. ${ }^{40}$ To each eigenvalue, there corresponds an eigenvector associated with a direction in phase space. In a Hamiltonian system with $N$ degrees of freedom, the $2 N$ eigenvalues must come in complex conjugate pairs which fall into one of the following types. (1) Pairs of imaginary $\lambda_{i}$ eigenvalues. Each pair signifies that the linearized motion in the vicinity of the critical point is oscillatory in the two corresponding directions and hence stable under small perturbations. This stability type is called stable or elliptic (E). (2) Pairs of real $\lambda_{i}$ eigenvalues. Each pair signifies that the motion in the 
vicinity is exponentially attracted to/repelled from the critical point in time, i.e., unstable in the two corresponding directions. This stability type is called unstable or hyperbolic $(\mathrm{H})$. (3) Quadruplets of complex eigenvalues $( \pm a \pm b i)$. This stability type is called mixed (M). ${ }^{10}$ In mixed stability, the solution contains both oscillating and exponential attraction/repulsion components in the subspace spanned by the four $\vec{V}_{i}$ corresponding to the quadruple $\lambda_{i}$. In two of the four directions the nearby trajectory spirals into the critical point, while in the other two directions it spirals out of the critical point. (4) Pairs of $\lambda_{i}=0$. This stability type is called degenerate. In this case, the linearized equations (42) become insufficient, and higher-order terms in the Taylor expansion are needed to evaluate the stability near a critical point. This situation does not arise for the acetylene bends and will not be considered further.

Specializing to $N=2$ as in (42), with none of the four $\lambda_{i}$ equal to zero, the possible types of linear stability are as follows: (a) two pairs of imaginary eigenvalues ( $a i,-a i, b i$, $-b i)$, bi-stable (EE); (b) two pairs of real eigenvalues $(a,-a$, $b,-b)$, bi-unstable $(\mathrm{HH})$; (c) one imaginary pair and one real pair $(a i,-a i, b,-b)$, stable-unstable (EH); (d) one complex quadruplet $(a+b i, a-b i,-a+b i,-a-b i)$, mixed (M). Here $a, b$ are positive real numbers.

For the reason described in IV.A, the linear stability of critical points A, B, C, and D in Figure 3 cannot be directly determined from (42) using the $\left(J_{a}, J_{b}, \psi_{a}, \psi_{b}\right)$ coordinates. Instead, the Hamiltonian is rewritten in a local-mode representation, in which points $A-D$ are no longer problematic. The stabilities of these critical points are then determined by stability matrixes written in the new action-angle variables, in a form equivalent to (42).

\section{Results}

The method of solution for the critical points is presented in section IV.A, resulting in the great circle conditions and the solutions for the action values on these circles listed in Table 1. In this section we discuss the meaning of these results.

For each of the four $\left(\psi_{a}, \psi_{b}\right)$ conditions on great circles given in $(33-36)$, there is a family of critical points. Each family corresponds to a new type of mode, applying the reasoning of section 3 as follows. Of the two cyclic angles $\theta_{a}, \theta_{b}$, the frequency of the latter

$$
\dot{\theta}_{b}=\frac{\partial H}{\partial K_{b}}
$$

is always zero when all the conditions assumed so far hold

$$
\sin \left[2 \psi_{a}\right]=\sin \left[2 \psi_{b}\right]=J_{b}=K_{b}=0
$$

leaving $\theta_{a}$ as the only variable that changes between 0 and $2 \pi$ with time. Each critical point therefore corresponds to a PO, i.e., a new anharmonic mode (more precisely, an infinitely degenerate family of POs because of the double degeneracy of the mode). The new modes are named local, orthogonal, precessional, and counter-rotator $(\mathbf{L}, \mathbf{O}, \mathbf{P}, \mathbf{C R})$ in accord with the shape of their corresponding Cartesian trajectories. Hereafter, we will use Trans, Cis, $\mathbf{L}, \mathbf{O}, \mathbf{P}$ and $\mathbf{C R}$ when referring to the critical points as such.

The $\mathbf{L}$ and $\mathbf{P}$ modes are planar motions as depicted previously in Figure 1 of the planar model of ref 8 . The $\mathbf{L}$ mode has the amplitude preferentially in one local $\mathrm{C}-\mathrm{C}-\mathrm{H}$ bend, while in the $\mathbf{P}$ mode the two $\mathrm{C}-\mathrm{C}-\mathrm{H}$ benders move with the same amplitude, but with a phase difference of $\pi / 2$. The $\mathbf{O}$ and $\mathbf{C R}$ modes are nonplanar. At $J_{a} / K_{a}=0$, the modes take their ideal forms. In the ideal $\mathbf{O}$ mode, the $\mathrm{C}-\mathrm{C}-\mathrm{H}$ benders move in

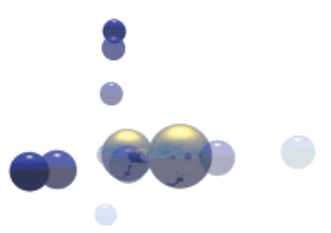

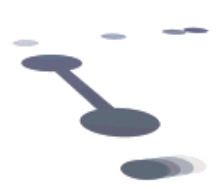

(a)

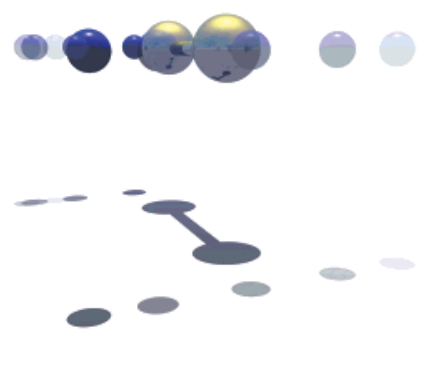

(c)

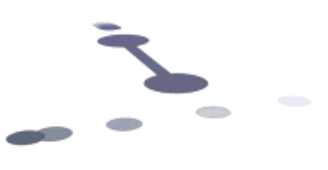

(b)
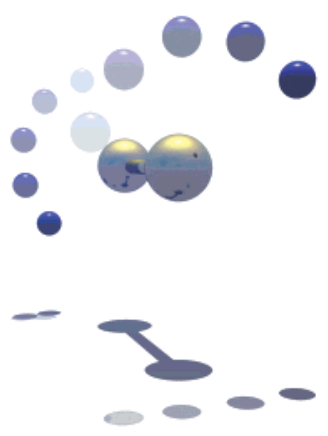

(d)
Figure 5. Time-lapse still frames from the animations in ref 42 of the new modes born in bifurcations. Panels a $-\mathrm{d}$ are the Local $\mathbf{L}$, Orthogonal $\mathbf{O}$, Precessional $\mathbf{P}$, and Counter-Rotator $\mathbf{C R}$ modes, respectively. The significance of the shadows in relation to the angle variable $\psi_{a}$ is discussed in the main text.

(W) Animations of the four modes in Quicktime format.

orthogonal planes, in phase with each other. The ideal CR has the hydrogens rotating in circles in opposite directions. To help visualize the modes, we converted the calculated trajectories into three-dimensional animations (Apple QuickTime format) with the software Bryce $4 .{ }^{41}$ These can be viewed at the website in ref 42, and also as Web Enhanced Objects linked to the online edition of this article. The animations give direct visual insight into the meaning of "new modes born in bifurcations". The modes are also depicted in time-lapse still frames in Figure 5.

The result that the critical points correspond to POs and therefore "new modes" is worthy of further comment. So far as we know, this has not previously been demonstrated in the case of the $l=0$ acetylene bends system. It is often taken for granted that bifurcations of normal modes in molecules will give "new modes". However, as the discussion in section 3 shows, this is not always the case: multiple polyad numbers can lead to higher-dimensional tori in bifurcations instead of POs. That POs are the case for the $l=0$ acetylene bends has been demonstrated mathematically with conditions (44). It is worth considering physically why it has to be this way. This is most easily seen in the local representation, as we now describe qualitatively, omitting the mathematical details. The condition $l=0$ means the molecular "shape" cannot have an overall rotation about the figure axis. A local representation condition analogous to (38) means that the shapes on the two ends of the molecule cannot twist with respect to each other. Together, these conditions imply that at the critical points, the local oscillators must move in POs along fixed ellipses, either with zero width, i.e., a line, as in the $\mathbf{L}, \mathbf{O}, \mathbf{P}$ modes, or in ellipses with finite width as in the $\mathbf{C R}$ mode. This is especially vivid in the animations of ref 42 . Section 8 briefly describes the situation 


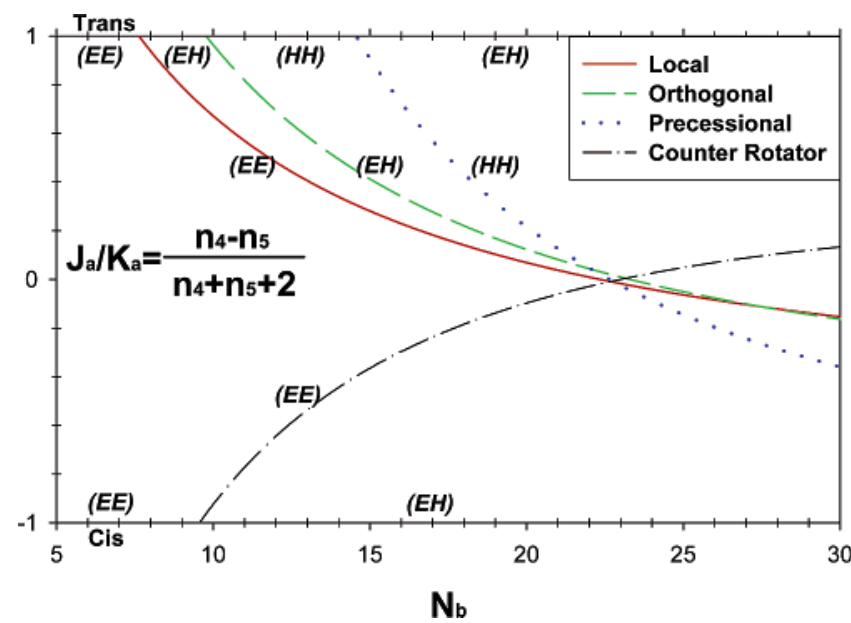

Figure 6. Bifurcation diagram from the critical points analysis, including the normal modes Trans, Cis, and four new families of critical points associated with new modes $\mathbf{L}, \mathbf{O}, \mathbf{P}, \mathbf{C R}$. Also shown is the stability of each critical point, including the normal modes before and after each bifurcation.

in the acetylene stretch-bends system where the critical points will not be limited to POs.

The angles $\psi_{a}$ and $\psi_{b}$ have a visual interpretation in the computer animations of ref 42 and the time-lapse still frames of Figure 5. By definition $\psi_{a}$ is the relative phase angle between the Trans and Cis modes, while $\psi_{b}$ can be interpreted (when $J_{b}=0$ as is the case for all the critical points discussed here) as the dihedral angle between the Trans and Cis planes. We recall that in section 4 , because of the dominant DD-I and $l$-resonances, all the new critical points are located at discrete $\left(\psi_{a}, \psi_{b}\right)$ values defined by the great circles on the respective single resonance phase spheres. With $\psi_{a}, \psi_{b}$ taking the discrete values in (33-36), the relative phase angle and the dihedral angle are locked at either 0 or $\pi / 2$. The association with $\psi_{a}$ is particularly evident in the animations but can also be discerned in the still frames of Figure 5. The projection on the ground plane (the oscillating "shadow") manifests the $\psi_{a}$ value at which the critical point is locked. In the four mode animations, $\mathbf{L}$ and $\mathbf{O}$ are locked at $\psi_{a}=0$ and have a corresponding asymmetry in the amplitudes of the shadows of the two $\mathrm{C}-\mathrm{C}-\mathrm{H}$ units. $\mathbf{P}$ and $\mathbf{C R}$ are locked at $\psi_{a}=\pi / 2$ and correspondingly have shadows of equal amplitude for the $\mathrm{C}-\mathrm{C}-\mathrm{H}$ units, bending out of phase by $\pi / 2$. (Again, this is more evident in the animations than the time-lapse Figure 5.) This phase relationship of $\mathbf{L}$ with $\mathbf{O}$ and of $\mathbf{P}$ with $\mathbf{C R}$ is essentially the result of the DD-I resonance. Identical phase relationships are seen in the planar single-resonance analysis ${ }^{8}$ of the DD-I coupling, with $\psi_{a}=0$ for the local mode and $\psi_{a}=\pi / 2$ for the precessional mode; see also the remarks in the final paragraph of this section on the mode stabilities in the planar model.

The diagram of the bifurcation "tree" of the bends system is obtained by plotting the critical points of Table 1 in action space, as shown in Figure 6 . The $x$-axis is the polyad number $N_{b}$, and the $y$-axis $J_{a} / K_{a}=\left(n_{4}-n_{5}\right) /\left(n_{4}+n_{5}+2\right)$ represents the relevant fraction of zero-order trans and cis bend action. The top of the $y$-axis therefore corresponds to Trans while the bottom corresponds to Cis. Each of the families has originated at some polyad action $N_{b}$ in a pitchfork bifurcation out of Trans or Cis (upper right and lower left corners of the squares in Figures $3,4)$. Immediately after each bifurcation, the new mode has $J_{a} /$ $K_{a}$ very close to the respective normal mode. The corresponding Cartesian trajectory is very much like the normal mode itself. Then as the polyad number increases, the new modes migrate toward $J_{a} / K_{a}=0$ (equal mixing between zero-order trans and cis action). As noted earlier, the new modes migrate from the corners along the diagonal in the pseudopotential diagrams in Figure 4; this constraint comes from the condition (38).

We now consider in detail the stability of each of the new modes and the changes in stability of the normal modes at the branch points of the bifurcation tree, using the methods of section IV.B. At each of the four normal mode bifurcations the stability of the normal mode changes when the new mode is born. The stability character is given by E (elliptical, or stable) or $\mathrm{H}$ (hyperbolic, or unstable) in each direction. At $N_{\mathrm{b}}=7.63$, Trans bifurcates to give the stable (EE) L, while itself changing from $(\mathrm{EE})$ to $(\mathrm{EH})$. Trans undergoes two more bifurcations at $N_{b}=9.77$ and 14.55. The new mode at $N_{b}=9.77$ is the $(\mathrm{EH})$ type $\mathbf{O}$ mode, the Trans now changing from $(\mathrm{EH})$ to $(\mathrm{HH})$. In the final Trans bifurcation at 14.55 the $(\mathrm{HH})$-type $\mathbf{P}$ mode is born, and Trans changes from $(\mathrm{HH})$ back to $(\mathrm{EH})$. Similarly, Cis bifurcates at $N_{b}=9.56$ to give the $\mathbf{C R}$ mode (EE), Cis itself changing from (EE) to $(\mathrm{EH})$ stability.

The stability character of these modes corresponds with the marks of classical structure on quantum wave functions investigated by Taylor and co-workers. ${ }^{17,18}$ (Further discussion of the classical investigation of refs 17 and 18 is found in section 7.) The bi-stable $\mathbf{L}$ and $\mathbf{C R}$ are surrounded by regions of invariant tori in phase space, around which are organized quantum states with clear nodal patterns and readily assigned quantum numbers. The $\mathbf{O}$ mode with $(\mathrm{EH})$ stability has distinguishable trajectories in coordinate space and slightly marks the quantum states. The bi-unstable $(\mathrm{HH}) \mathbf{P}$ mode is completely engulfed in chaos in surfaces of section and appears not to be associated with assignable quantum numbers in wave functions.

It should be noted that Rose and Kellman ${ }^{8}$ performed a precursor of the present study, a bifurcation analysis of the fitting Hamiltonian using a simplified essentially planar model with just the DD-I coupling. They found that between $N_{b}=6-8$ the trans-bend becomes unstable and a stable local mode is born; at $N_{b} \approx 16$ the cis-bend becomes unstable and a stable precessional mode is born. This compares with the present threeresonance study where $\mathbf{L}$ is again found to be bi-stable but $\mathbf{P}$ is bi-unstable. (See the remarks in the concluding section on the "doubling" of the planar local and precessional modes to $\mathbf{L}, \mathbf{O}$ and $\mathbf{P}, \mathbf{C R}$ in three dimensions.)

\section{Comparison of Effective Hamiltonians}

In the next two sections, we evaluate the picture of acetylene bend dynamics that emerges from comparison of the present with various other treatments of the problem. In this section we compare bifurcation analysis of other versions of the effective Hamiltonian than the one analyzed above; in the next section we compare results from approaches other than bifurcation analysis of effective Hamiltonians.

The bifurcation analysis depicted in Figure 6 uses what we believe is the best empirically fit Hamiltonian for the pure bending spectra of acetylene. It is important to confront these results with those obtained from other available versions of the effective Hamiltonian. These include fitting Hamiltonians which attempt to merge the results of dispersed fluorescence (high bend excitation) and absorption (high stretch excitation) spectroscopy and an effective Hamiltonian obtained from a potential energy surface by means of perturbation theory.

Figure 7 shows the results of the bifurcation analysis on six such Hamiltonians. The first (a) is the same Hamiltonian of ref 15 as we used for Figure 6, except that the high-order 
(a)

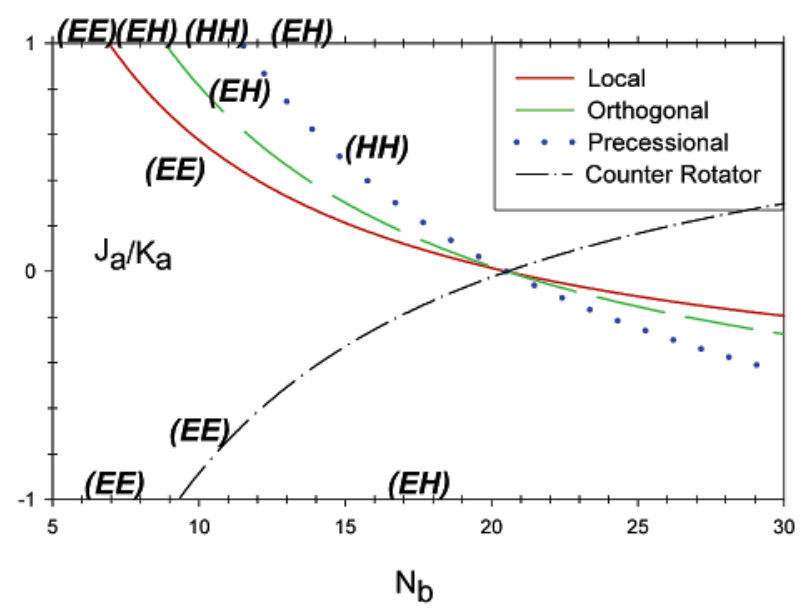

(c)

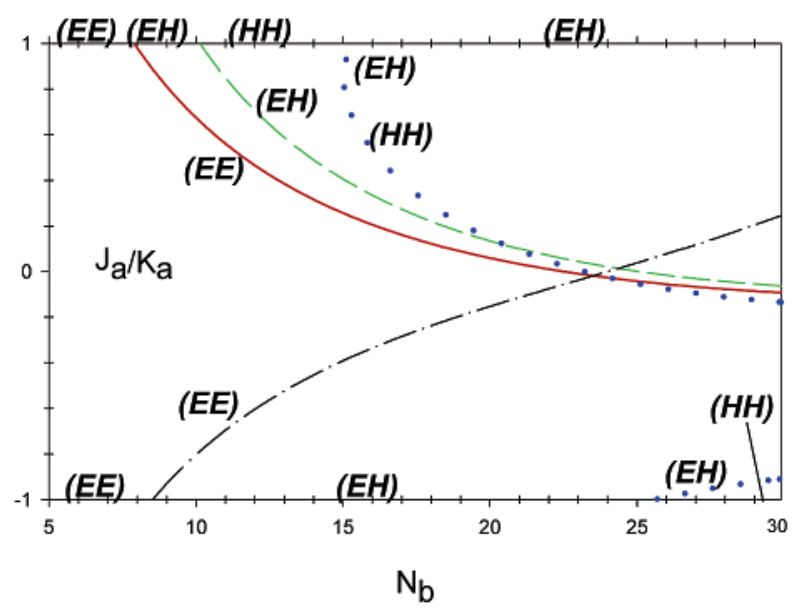

(e)

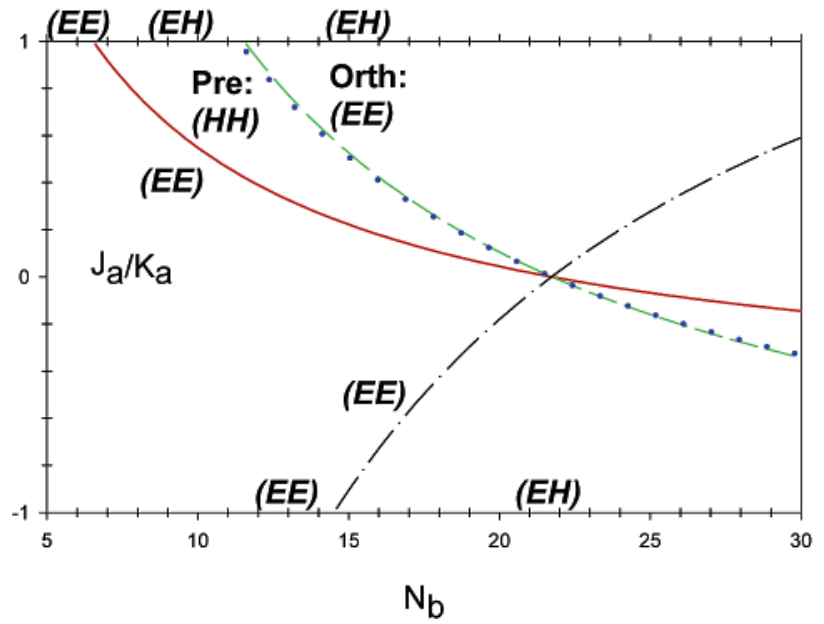

(b)

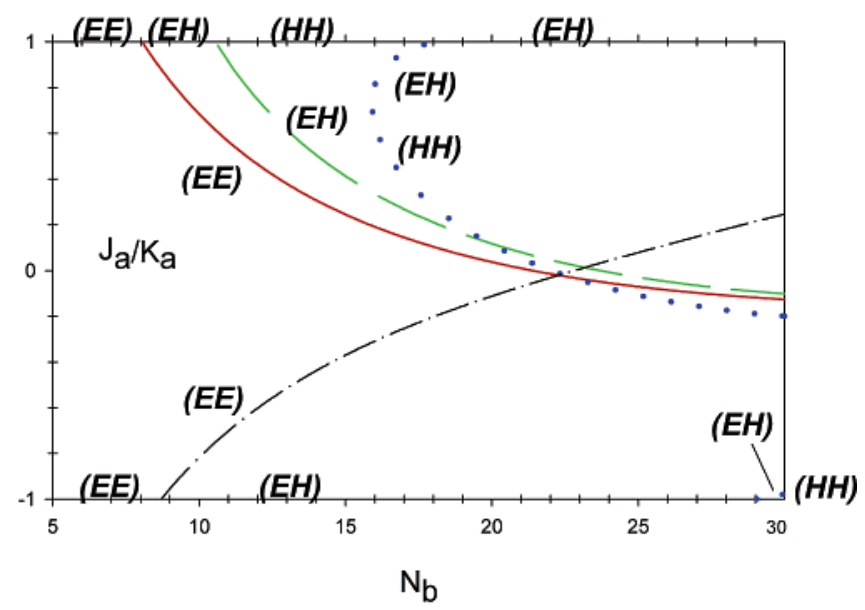

(d)

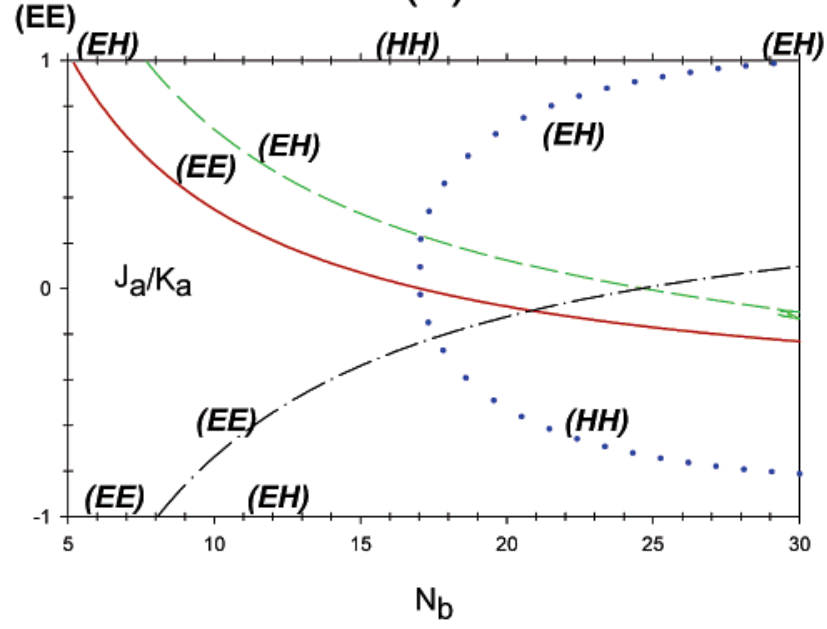

(f)

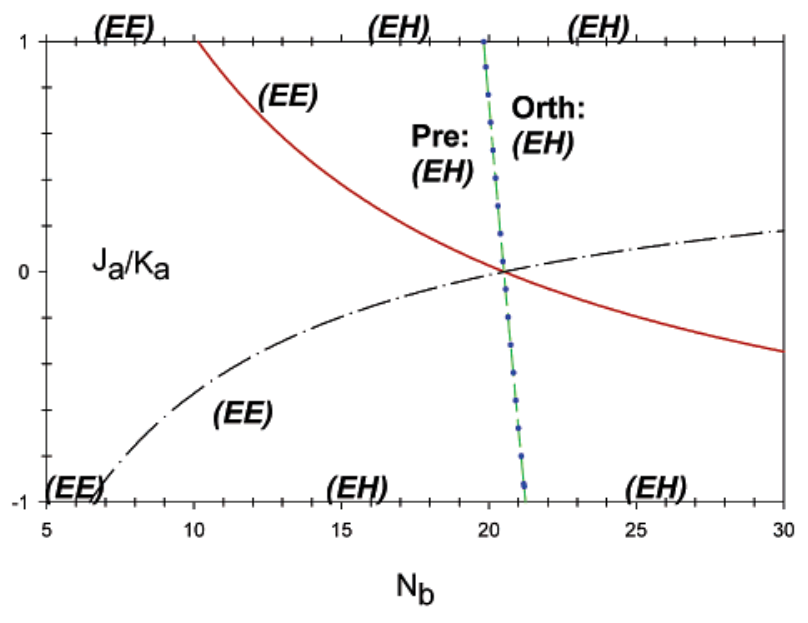

Figure 7. Bifurcation diagrams for effective Hamiltonians with alternative parameter sets, as described in section 6. Panels a-d are for experimentbased effective Hamiltonians similar to the Hamiltonian used in Figure 6. Panel e is for a Hamiltonian obtained by Sibert and McCoy ${ }^{43 b}$ via perturbation theory from a potential energy surface; panel $\mathrm{f}$ is for a "minimal" Hamiltonian devised here for two coupled local bend modes.

coefficients $y_{i j k}, r_{445}$, and $r_{455}$ are ignored. The second Hamiltonian (b) is a more recent refinement ${ }^{24}$ based on that of ref 15: the parameters are slightly modified and there is an additional fourth-order term $z_{4455} n_{4}^{2} n_{5}^{2}$ in (4). This Hamiltonian was given in Table 2 of ref 24. (We used the earlier Hamiltonian of ref 15 in our bifurcation analysis because it fits higher energy experimental levels, so we believe it more accurately reflects highly excited bending dynamics.) The third Hamiltonian (c) was developed by Hoshina et al. ${ }^{24}$ by combining available stretch-bend data from dispersed fluorescence and absorption spectra. The fourth Hamiltonian (d) is the fit of Herman and co-workers ${ }^{33}$ of stretch-bend spectra, constructed to reflect data 
from a variety of spectral techniques. This Hamiltonian does not have the pure bending cubic coefficients, nor does it contain the DD-II resonance term in (3). We adapted the stretch-bend Hamiltonian in (c) and (d) for pure bend spectra by setting all the stretch quantum numbers to zero.

Effective Hamiltonians very similar to a spectroscopic fitting Hamiltonian can be obtained from quantum potential energy surfaces by means of perturbation theory. ${ }^{22,31,43,44}$ McCoy and Sibert ${ }^{43}$ have produced the only effective Hamiltonian acetylene bends that we know of obtained in this way. The bifurcation diagram obtained for this Hamiltonian (with minor correction, see ref 45) using the critical points method is shown in Figure 7e. (As described in the next section, McCoy and Sibert obtained the local mode for this Hamiltonian using critical points conditions.)

The final Hamiltonian is a "minimal" Hamiltonian consisting of two doubly degenerate local bend oscillators with only symmetry-adapted 1:1 couplings. The Hamiltonian is truncated at terms second-order in the quantum numbers, with parameters taken from the bends fitting Hamiltonian transformed to the local mode representation. The reason to consider such a model is that such a Hamiltonian in a two-mode version ${ }^{5,6}$ is a paradigm for understanding the coexistence of local and normal modes in coupled stretch systems such as $\mathrm{H}_{2} \mathrm{O}$. The bifurcation diagram for the acetylene bends minimal Hamiltonian is shown in Figure $7 f$.

The most striking thing about the bifurcation diagrams in Figures 6 and 7 is their overall consistency. All the Hamiltonians give the same basic feature of four new modes born in bifurcations. Panels $\mathrm{a}-\mathrm{c}$ of Figure 7 are all very similar to Figure 6. In parts b and c of Figure 7 the $\mathbf{P}$ branch is "bent" into a tangent bifurcation, but one branch of this joins Trans as in Figures 6 and 7a. Hence, this does not constitute a separate bifurcation tree, unlike a tangent bifurcation found in HCP. ${ }^{11}$ In Figure $7 d$ based on the early 1995 fit, ${ }^{33}$ the $\mathbf{P}$ branch is more severely bent. This shows the importance of progressive refinements of the fitting Hamiltonian. In all five cases Figures 6 and $7 \mathrm{a}-\mathrm{d}$, the normal mode from which each branch originates and the stability of each branch match.

In the last two panels Figure 7e,f, the differences are more pronounced. This is hardly surprising in that the Hamiltonians described above for these panels were never expected to closely match very highly excited experimental data. In Figure 7e the perturbation Hamiltonian switches the stability of $\mathbf{O}$ from the previous figures. The most surprising thing about Figure $7 \mathrm{f}$ for the minimal Hamiltonian is how closely it mirrors the results from the refined fitting Hamiltonians, given the extreme simplicity of the minimal Hamiltonian.

\section{Comparison with Other Approaches}

Next, we compare results from several other approaches with the results of the preceding bifurcation analysis.

Using different approaches, McCoy and Sibert ${ }^{43}$ and Rose and Kellman ${ }^{8}$ verified the existence of a suspected normal-tolocal modes transition in acetylene bending vibrations. ${ }^{39,46}$ As noted at the end of section V, Rose and Kellman ${ }^{8}$ performed a precursor of the present study, a bifurcation analysis of the fitting Hamiltonian using a simplified model with just the DD-I coupling. As discussed in the preceding section, McCoy and Sibert performed a van Vleck perturbation analysis of an acetylene potential surface to get an effective Hamiltonian, whose full critical points bifurcation analysis we performed and discussed in Figure 7e. McCoy and Sibert analyzed this Hamiltonian using critical points conditions as well as wave function plots and obtained the $\mathbf{L}$ mode. Our bifurcation analysis showed that this Hamiltonian also contains information about the $\mathbf{O}, \mathbf{P}$, and $\mathbf{C R}$ modes. We would like to emphasize the great potential utility of applying critical points analysis to effective Hamiltonians obtained from potential surfaces via perturbation theory. This is an alternative to running trajectories on surfaces and searching numerically for bifurcations. In fact, we believe that comparison of bifurcation behavior of potential surfaces with experimentally derived analysis like Figures 6 and 7 is an extremely valuable way to assess the validity of surfaces.

The classical version of the fitting Hamiltonian used in this paper was studied previously by Taylor and co-workers ${ }^{17,18}$ in an investigation of surfaces of section generated by numerically integrating Hamilton's equations. A "family tree" of fixed points as they appear on surfaces of sections was constructed for two different polyads $N_{b}=16,22$ by following the fixed points as the energy is varied at fixed value of the polyad number. This is a significant difference from the present study, where we have followed critical points as the polyad number is varied. In the surfaces of section at fixed polyad numbers of refs 17 and 18, points appear as fixed points but actually are not in general POs (1-tori) in the full phase space, but rather 2-tori. (They are 2-tori when both cyclic frequencies $\dot{\theta}_{a}, \dot{\theta}_{b} \neq 0$, and POs for the special condition $\dot{\theta}_{b}=0$, i.e., the critical points determined by the present methodology. This is similar to the observations of $\mathrm{Lu}$ and Kellman ${ }^{9}$ in surfaces of section for $\mathrm{H}_{2} \mathrm{O}$.)

The cardinal finding of refs 17 and 18 is the emergence of new phase space structures at higher polyads, particularly a local and a counter rotator family, around which the bottom and top energy eigenfunctions in the polyad localize, respectively. Classically, these families are 2-tori localized around the $\mathbf{L}$ and CR modes. There is another structure which Taylor and coworkers call the " $M_{2}$ mode", which appears to be related to our O mode with (EH) stability. The Cartesian trajectories of $M_{2}$ (see Figure 3 of ref 17 and Figure 1 of ref 18) appear to be motion on 2-tori which have the same general shape as that of $\mathbf{O}$, with the two $\mathrm{C}-\mathrm{H}$ benders perpendicular but in phase with each other. Their motion is also stable in the radial direction and precesses significantly in the angular direction, consistent with our finding of $(\mathrm{EH})$ stability for $\mathbf{O}$.

We found that the $\mathbf{P}$ critical point is bi-unstable $(\mathrm{HH})$ and so expected to be surrounded by chaos. Indeed, this is how the surrounding region appears in the work of Taylor and coworkers in surfaces of section (see Figure 1 of ref 18, sixth column, left side of the second and third panel). Since their method locates fixed points surrounded by a stable region, they did not observe the $\mathbf{P}$ mode.

Farantos and co-workers have performed a numerical PO search on the six-dimensional potential energy surface of $\mathrm{C}_{2} \mathrm{H}_{2} \cdot{ }^{47}$ A reexamination of the results ${ }^{48}$ confirmed the bifurcation of a local-mode PO out of the trans bend and a counter-rotator mode out of the cis bend. A more recent $a b$ initio potential energy surface $^{49}$ has yielded the same results in the quantum wave functions, this time with quantitative agreement with experimental and theoretical results. These studies provide important independent evidence for the birth of stable modes in bifurcations, with pronounced consequences for the quantum states.

Further independent evidence comes from distinct methods involving correlation diagrams using the quantum version of the effective fitting Hamiltonian. Rose and Kellman ${ }^{50}$ found evidence for the stable local bend and what is now recognized as the stable counter-rotator mode, using assignments based on notions later developed into a dressed-basis computational 
approach. ${ }^{51}$ Semparithi and Keshavamurthy ${ }^{52}$ found both modes using a derivative-based method of analyzing the correlation diagram.

$\mathrm{Yu}$ and $\mathrm{Wu}^{53}$ have performed a coset semiclassical analysis on the fitting Hamiltonian and confirmed the following general trend: destabilization of trans and cis normal modes between $N_{b}=6$ and 14 and gradual migration of new modes with "uneven distribution between the two normal mode characters" in phase space, until they reach the local/counter-rotator type at polyad 22 .

Using Lie algebraic methods, Champion et al. ${ }^{54}$ deduced the existence of 15 possible types of ideal bending modes for a Hamiltonian with the form of $\mathrm{H}$ in (18), without regard to the parameters. Their examination of the 144 eigenstates in the $N_{b}$ $=22$ polyad showed that many of them can be approximately assigned to several of these 15 types. The majority of states at the bottom and top of the polyad in energy are of $\mathbf{L}$ and $\mathbf{C R}$ type, consistent with the findings of the present work.

This section and the preceding demonstrate broad consistency in the picture of acetylene bend dynamics that has emerged in recent years. In particular, comparisons of bifurcation analysis of several different versions of the effective Hamiltonian, as well as independent results of other approaches, clearly show the existence of stable $\mathbf{L}$ and $\mathbf{C R}$ modes. The finding of additional $\mathbf{O}$ and $\mathbf{P}$ modes is a new result of the present study. These appear in all of the bifurcation studies of effective Hamiltonians but may not be clear-cut in other approaches because of their instability.

\section{Discussion and Conclusions}

The bifurcation analysis using the analytical critical points method has achieved its principal goals. The new modes born in bifurcations of the normal modes have been accounted for and a rational account of their stability character obtained. A global view of the bending dynamics has been achieved from lowest to highest energies of known spectra. Comparison of Hamiltonians from different types of spectroscopy shows consistency of the dynamical picture as represented in bifurcation behavior. Comparison of the bifurcation analysis with other approaches shows consistency in the comprehensive picture that has emerged in recent years. There is widespread agreement on the existence of what we have labeled stable $\mathbf{L}$ and $\mathbf{C R}$ modes. There are more subtle differences in various studies regarding the unstable $\mathbf{O}$ and $\mathbf{P}$ modes.

The observation that the new modes lie at special angles in phase space, i.e., on great circles on the phase spheres, has been accounted for in terms of the great circles conditions on the two major resonance couplings, the $l$-resonance and the Darling-Dennison I and the comparative weakness of the Darling-Dennison II. It remains an open question whether this property of the strongest individual resonances constraining the location of critical points will hold in general, especially for the full stretch-bend system, which has a large number of independent couplings.

We have found that all the new modes are part of a single "evolutionary tree" of the original normal modes and new modes born in their bifurcations. This contrasts with $\mathrm{HCP},{ }^{11}$ where an "isomerization mode" is born independently in a saddle-node bifurcation, rather than branching directly from one of the normal modes. Furthermore, in the acetylene bends, the modes born in bifurcations of the normal modes do not themselves undergo subsequent bifurcations. This contrasts with $\mathrm{H}_{2} \mathrm{O}$. ${ }^{9}, 10,14$

We now have a rather complete picture of the bifurcation behavior of the $l=0$ acetylene bends system as inferred from a spectroscopic Hamiltonian that goes up to near the isomerization barrier. Several questions suggest themselves for future investigation.

In this investigation of $l=0$ acetylene bends, as in previous studies of other molecules, it has been found that the normal modes always bifurcate to give 1-tori i.e., POs ("new modes born in bifurcations"). As emphasized in section 3, this is by no means always the case. Bifurcations to higher dimensional tori are expected in general whenever there are multiple polyad numbers. Here there are polyad numbers $N_{b}$ and $l$, and the existence of modes born in bifurcations cannot be taken for granted. The special case $l=0$ in fact leads to bifurcations to 1 -tori, as can be shown by symmetry considerations. For $l \neq 0$, the critical points will correspond to 2-tori. This again is not unexpected; the extra dimension of the torus corresponds to precession of orbits with respect to the symmetry axis, as expected with anharmonic bend modes. However, in systems such as full stretch-bend dynamics of acetylene, there is a third polyad number; see (1) and refs 20,21 , and 33. The analysis of the bifurcation behavior in such a system is a challenging open problem.

The analytical critical points method gives an algorithm to obtain the bifurcation behavior of the spectroscopic Hamiltonian. It can still be asked if there is some a priori way to predict why there should be precisely four modes born in bifurcations. There are two phase space spheres, each associated with one of the dominant resonance couplings, and each with two great circles, with four new modes $\mathbf{L}, \mathbf{O}, \mathbf{P}, \mathbf{C R}$ arising in bifurcations on these great circles. This doubling of the two new modes $\mathbf{L}, \mathbf{P}$ found on the single sphere of the planar acetylene bends model, ${ }^{8}$ as well as the phase-locking relations between $\mathbf{L}$ and $\mathbf{O}$ and between $\mathbf{P}$ and $\mathbf{C R}$ discussed in section 5, suggests that there is some at least approximate organizing principle for the threedimensional bend system that remains to be understood. We are currently investigating this.

A related issue is whether one can obtain a formal classification of the entire phase space structure, not just the critical points themselves. In single-resonance systems, this is easily accomplished, with the organization defined by the locations of stable fixed points, together with the unstable fixed points joined by separatrices (stable and unstable manifolds). For example, for a pair of local modes coupled by a 1:1 resonance, the phase space divides into local and normal mode regions, as demonstrated comprehensively with the catastrophe map; ${ }^{6}$ for the more general Darling-Dennison Hamiltonian for a pair of stretches or a planar bends model, the slightly more complicated classification is also completely known. ${ }^{8} \mathrm{~A}$ similar classification is desirable for higher-dimensional systems; the analytical critical points analysis here is the first step.

It is noteworthy that each bi-stable normal mode spawns one set of bi-stable modes: the Trans gives $\mathbf{L}$; the $\mathbf{C i s}$ gives $\mathbf{C R}$. After their birth in a bifurcation, $\mathbf{L}$ lies at the bottom energy end of each polyad; $\mathbf{C R}$ lies at the top. $\mathbf{L}$ constitutes the isomerization "reaction mode" for the acetylene-vinylidene isomerization. An isomerization mode lying at the bottom of each polyad has also been observed in $\mathrm{HCP} ;{ }^{11}$ this observation is suggestively consistent with the notion of a minimum energy reaction path. Whether these are instances of a general property of isomerization modes in bifurcating molecular vibration systems appears to be an open question.

As emphasized in section 7, critical points analysis of effective Hamiltonians obtained via perturbation theory provides a novel and stringent way of testing potential surfaces against experiment. This involves comparison with the bifurcation 
behavior of effective Hamiltonians obtained from fitting spectra. It is an alternative to running trajectories on surfaces and searching numerically for bifurcations. In particular, for recent potentials ${ }^{49}$ developed for high excitations of acetylene, perturbation treatment followed by bifurcation analysis and comparison with experimentally based results such as Figures 6 and 7 would be most valuable.

The problems of $l \neq 0$ dynamics, rotation-vibration interaction, and the full stretch-bend dynamics are ripe for analysis by the critical points method. Extension of the spectroscopic Hamiltonian to encompass isomerization dynamics, with multiple wells for the acetylene and vinylidene forms, and motion above the isomerization, is an outstanding problem. Recent works ${ }^{55,56}$ are steps in this direction. In this connection, it appears desirable to establish a link between the present developments in analysis of effective Hamiltonians and recent developments using a phase space approach to transition state theory. ${ }^{57}$

As a final remark, this paper has used fits of very complex highly excited vibrational frequency domain spectra to decode information about invariants (new modes born in bifurcations) that can reasonably be considered "dynamical" in nature. An open question is to what extent the phenomena uncovered here can be accessed in time-domain spectroscopy and control. For example, the excitation and subsequent time evolution of coherent states along the new modes discussed here would be of great interest.

Acknowledgment. This work was supported by the U.S. Department of Energy Basic Energy Sciences program under Contract DE-FG02-05ER15634.

\section{References and Notes}

(1) Kellman, M. E. Annu. Rev. Phys. Chem. 1995,46, 395-421.

(2) Kellman, M. E. In Molecular Dynamics and Spectroscopy by Stimulated Emission Pumping; Dai, H.-L., Field, R. W., Eds.; Advanced Series in Physical Chemistry; World Scientific: Singapore, 1995; Vol. 4, pp 943-997.

(3) Kellman, M. E. Internal Molecular Motions. In Encyclopedia of Chemical Physics and Physical Chemistry; Moore, J. H., Spencer, N. D., Eds.; Institute of Physics: London, 2001; Vol. 1.

(4) Kellman, M. E.; Lynch, E. D. J. Chem. Phys. 1986, 85, 58555869.

(5) Xiao, L.; Kellman, M. E. J. Chem. Phys. 1989, 90, 6086-6098.

(6) Xiao, L.; Kellman, M. E. J. Chem. Phys. 1990, 93, 5805-5820.

(7) Svitak, J.; Li, Z.; Rose, J.; Kellman, M. E. J. Chem. Phys. 1995, $102,4340-4354$ 10754 .

(8) Rose, J. P.; Kellman, M. E. J. Chem. Phys. 1996, 105, 10743-

(9) Lu, Z.-M.; Kellman, M. E. Chem. Phys. Lett. 1995, 247, 195202.

(10) Lu, Z.-M.; Kellman, M. E. J. Chem. Phys. 1997, 107, 1-15.

(11) Joyeux, M.; Sugny, D.; Tyng, V.; Kellman, M. E.; Ishikawa, H.;

Field, R. W.; Beck, C.; Schinke, R. J. Chem. Phys. 2000, 112, 4162-4172.

(12) Zhou, C.; Xie, D.; Chen, R.; Yan, G.; Guo, H.; Tyng, V.; Kellman,

M. E. Spectrochim. Acta, A 2002, 58, 727-746.

(13) Svitak, J. F.; Tyng, V.; Kellman, M. E. J. Phys. Chem. A 2002, 106, 10797-10805. 179 .

(14) Keshavamurthy, S.; Ezra, G. S. J. Chem. Phys. 1997, 107, 156-

(15) Jacobson, M. P.; O'Brien, J. P.; Silbey, R. J.; Field, R. W. J. Chem. Phys. 1998, 109, 121-133.

(16) Kiefer, J. H.; Mudipalli, P. S.; Wagner A. F.; Harding, L. J. Chem. Phys. 1996, 105, 8075-8096.

(17) Jacobson, M. P.; Jung, C.; Taylor, H. S.; Field, R. W. J. Chem. Phys. 1999, 111, 600-618.

(18) Jung, C.; Taylor, H. S.; Jacobson, M. P. J. Phys. Chem. A 2001, 105, 681-693.
(19) El Idrissi, M. I.; Liévin, J.; Campargue, A.; Herman, M. J. Chem. Phys. 1999, 110, 2074-2086.

(20) Kellman, M. E. J. Chem. Phys. 1990, 93, 6630-6635.

(21) Kellman, M. E.; Chen, G. J. Chem. Phys. 1991, 95, 8671-8672.

(22) Fried, L. E.; Ezra, G. S. J. Chem. Phys. 1987, 86, 6270-6282.

(23) Jacobson, M. P. Ph.D. Thesis, Massachusetts Institute of Technology, Cambridge, MA, 1999.

(24) Hoshina, K.; Iwasaki, A.; Yamanouchi, K.; Jacobson, M. P.; Field, R. W. J. Chem. Phys. 2001, 114, 7424-7442.

(25) Cohen-Tannoudji, C.; Diu, B.; Laloë, F. Quantum Mechanics; John Wiley \& Sons: New York, 1977; Vol. 1, Complements to Chapter VI, $D_{\text {VI }}$, pp $727-741$.

(26) Heisenberg, W. Z. Phyz. 1925, 33, 879; translated in Sources of Quantum Mechanics; van der Waerden, B. L., Ed.; Dover: New York, 1967.

(27) Child, M. S. Semiclassical Mechanics with Molecular Applications; Clarendon Press: Oxford, 1991; Chapter 4.2.

(28) Clark, A. P.; Dickinson, A. S.; Richards, D. Adv. Chem. Phys. 1977, $36,63-139$.

(29) Joyeux, M.; Farantos, S. C.; Schinke, R. J. Phys. Chem. A 2002, $106,5407-5421$.

(30) Bredenbeck, J.; Beck, C.; Schinke, R.; Koput, J.; Stamatiadis, S.; Farantos, S. C.; Joyeux, M. J. Chem. Phys. 2000, 112, 8855-8865.

(31) Jost, R.; Joyeux, M.; Skokov, S.; Bowman, J. J. Chem. Phys. 1999 $111,6807-6820$

(32) Rose, J. P.; Kellman, M. E. J. Chem. Phys. 1996, 105, 73487363.

(33) Abbouti Temsamani, M.; Herman, M. J. Chem. Phys. 1995, 102, 6371-6384.

(34) Eilbeck, J. C. In Davydov's Soliton Revisited: self-trapping of vibrational energy in protein; Christiansen, P. L., Scott, A. C. Eds.; NATO Advanced Research Workshop on Self-Trapping of Vibrational Energy in Protein; Plenum: New York, 1990.

(35) Bernstein, L.; Eilbeck, J. C.; Scott, A. C. Nonlinearity 1990 3, $293-$ 323. 4.

(36) Kellman, M. E.; Tyng, V. Phys. Rev. A 2002, 66, 13602/1-13602/

(37) Ding, X. Ph.D. Thesis, University of Oregon, Eugene, OR, 2004 URL: http://darkwing.uoregon.edu/ meklab/DingThesis/index.html

(38) Svitak, J. F. Ph.D. Thesis, University of Oregon, Eugene, OR, 1998

(39) Lehmann, K. K. J. Chem. Phys. 1992, 96, 8117-8119.

(40) Tabor, M. Chaos and Integrability in Nonlinear Dynamics; John Wiley \& Sons: New York, 1989.

(41) DAZ Productions Inc. URL: http://bryce.daz3d.com/

(42) URL: http://darkwing.uoregon.edu/ meklab/.

(43) (a) McCoy, A. B.; Sibert, E. L., III J. Chem. Phys. 1996, 105, 459468. (b) Sibert, E. L., III; McCoy, A. B. J. Chem. Phys. 1996, 105, 469478.

(44) Tyuterev, V. G. In Symmetry and Perturbation Theory; Abenda, S., Gaeta, G., Walcher, S., Eds.; Proceedings of the International Conference on SPT 2002; World Scientific: River Edge, NJ, 2002.

(45) Due to a misprint, the three coupling coefficients $\left(s_{45}, r_{45}\right.$, and $\left.t_{45}\right)$ in Table 1 of ref $43 \mathrm{~b}$ must be multiplied by a factor of 2 (E. L. Sibert, private communication).

(46) Jonas, D. M.; Solina, S. A. B.; Rajaram, B.; Silbey, R. J.; Field, R. W.; Yamanouchi, K.; Tsuchiya, S. J. Chem. Phys. 1993, 99, 7350-7370.

(47) Prosmiti, R.; Farantos, S. C. J. Chem. Phys. 1995, 103, 32993314.

(48) Prosmiti, R.; Farantos, S. C. J. Chem. Phys., 2003, 118, 82758280 .

(49) Xu, D.; Guo, H.; Zou, S.; Bowman, J. M. Chem. Phys. Lett. 2003, $377,582-588$

(50) Rose, J. P.; Kellman, M. E. J. Phys. Chem. A. 2000, 104, 1047110481

(51) Kellman, M. E.; Dow, M. W.; Tyng, V. J. Chem. Phys. 2003, 118, 9519-9527.

(52) Semparithi, A.; Keshavamurthy, S. Chem. Phys. Lett. 2004, 395 327-334.

(53) Yu, J.; Wu, G. J. Chem. Phys. 2000, 113, 647-652.

(54) Champion, J.-M.; Abbouti Temsamani, M.; Oss, S. Phys. Chem. Commun. (RSC online journal), 2000, article number 2.

(55) Yang, S.; Tyng, V.; Kellman, M. E. J. Phys. Chem. A 2003, 107 $8345-8354$

(56) Jacobson, M. P.; Child, M. S. J. Chem. Phys. 2001, 114, 250261.

(57) Uzer, T.; Jaffé, C.; Palacián, J.; Yanguas, P.; Wiggins, S. Nonlinearity 2002, 15, 957-992. 